\title{
$\frac{11}{6,9}, 968 \delta 0$ \\ 6 SANDIA REPORT
}

SAND96-1133 - UC-706

Unlimited Release

Printed May 1996

\section{Fiber Optic Hydrogen Sensor}

\author{
M. A. Butler, R. Sanchez, G. R. Dulleck
}

\section{Prepared by}

Sandia National Laboratories

Albuquerque, New Mexico 87185 and Livermore, California 94550

for the United States Department of Energy

under Contract DE-AC04-94AL85000

Approved for public release; distribution is unlimited. 
Issued by Sandia National Laboratories, operated for the United States Department of Energy by Sandia Corporation.

NOTICE: This report was prepared as an account of work sponsored by an agency of the United States Government. Neither the United States Government nor any agency thereof, nor any of their employees, nor any of their contractors, subcontractors, or their employees, makes any warranty, express or implied, or assumes any legal liability or responsibility for the accuracy, completeness, or usefulness of any information, apparatus, product, or process disclosed, or represents that its use would not infringe privately owned rights. Reference herein to any specific commercial product, process, or service by trade name, trademark, manufacturer, or otherwise, does not necessarily constitute or imply its endorsement, recommendation, or favoring by the United States Government, any agency thereof or any of their contractors or subcontractors. The views and opinions expressed herein do not necessarily state or reflect those of the United States Government, any agency thereof or any of their contractors.

Printed in the United States of America. This report has been reproduced directly from the best available copy.

Available to DOE and DOE contractors from

Office of Scientific and Technical Information

PO Box 62

Oak Ridge, TN 37831

Prices available from (615) 576-8401, FTS 626-8401

Available to the public from

National Technical Information Service

US Department of Commerce

5285 Port Royal Rd

Springfield, VA 22161

NTIS price codes

Printed copy: A03

Microfiche copy: A01 
SAND96-1133

Unlimited Release

Printed May 1996
Distribution

Category UC-706

\title{
Fiber Optic Hydrogen Sensor
}

\author{
M. A. Butler and R. Sanchez \\ Microsensor Research and Development Department \\ \& \\ G. R. Dulleck \\ Optoelectronics Applications Department \\ Sandia National Laboratories \\ Albuquerque, NM 87185
}

\begin{abstract}
This report covers the development of fiber optic hydrogen and temperature sensors for monitoring dissolved hydrogen gas in transformer bil. The concentration of hydrogen gas is a measure of the corona and spark discharge within the transformer and reflects the state of health of the transformer. Key features of the instrument include use of palladium alloys to enhance hydrogen sensitivity, a microprocessor controlled instrument with RS-232, liquid crystal readout, and 4-20 ma. current loop interfaces. Calibration data for both sensors can be down loaded to the instrument through the RS-232 interface. This project was supported by the Technology Transfer Initiative in collaboration with J. W. Harley, Inc. through the mechanism of a cooperative research and development agreement (CRADA).
\end{abstract}

\section{MASTER}


TABLE OF CONTENTS

\section{PAGE}

I. Introduction

3

II. Science of the hydrogen sensing coating. 5

III. Temperature sensing. 10

IV. Engineering the sensing elements. 12

V. Instrument design and development. 17

VI. Measurements in transformer oil. 26

$\begin{array}{ll}\text { VII. Commercialization Potential } & 28\end{array}$

VIII. Summary. $\quad 30$

IX. Acknowledgments. $\quad 30$

$\begin{array}{ll}\text { References. } & 31\end{array}$

$\begin{array}{ll}\text { Appendix A } & 32\end{array}$ 


\section{Introduction}

The goal of the CRADA as initiated was to explore Sandia's micromirror fiber optic sensing technology to see if it could be applied to a problem in the electrical utility industry. Specifically a goal was to the detection of dissolved hydrogen gas in the oil in large power transformers. Corona discharge inside the transformers decomposes the oil and other organic components in the transformer to produce the hydrogen. Thus the concentration of hydrogen in the oil is a measure of breakdown activity within the transformer and thus it's state of health. Since a rapid increase in hydrogen concentration can be an indicator of imminent failure, real time monitoring is required. Because of the large electric fields inside these transformers, wires cannot be used, making this the ideal environment for the application of a fiber optic hydrogen sensor.

Fiber optic hydrogen sensing utilizes the interaction of hydrogen with palladium alloys to reversibly form a hydride that has different optical properties than the pure metal. This change is observed by coating the material on the end of an optical fiber and measuring the reflectivity of the light transmitted through the fiber. Before this CRADA the hydrogen sensing concept had been demonstrated 1,2 using pure palladium metal. However, the demonstrated sensitivity was not as good as required for the transformer application, the sensor had not been demonstrated in liquids, and the phenomenon had only been demonstrated in the lab. Thus the approach of the CRADA was to explore palladium alloy compositions for improving the sensitivity, demonstrate effective performance of the hydrogen sensor in transformer oil, and to explore environmental effects on the sensor such as temperature, fiber losses, and other gases.

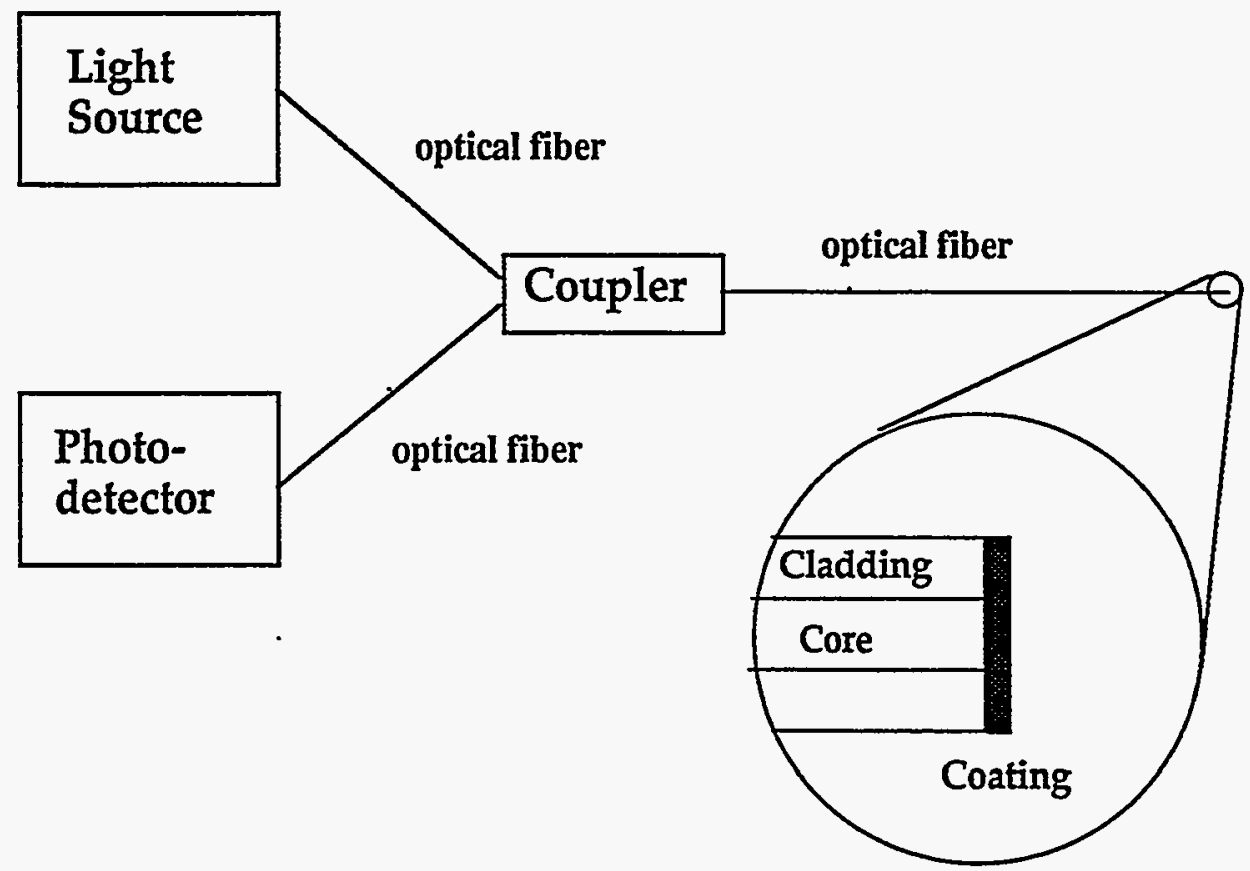

Fig. 1 Schematic of the micromirror chemical sensor. The light source is an $860 \mathrm{~nm}$ LED modulated at $10 \mathrm{kHz}$ and the photodetector is a silicon photodiode with synchronous detection of the signal. All fiber is multi-mode with $50 \mu \mathrm{m} / 125 \mu \mathrm{m}$ core/cladding diameters.

The basic sensor configuration is our "micromirror" chemical sensor configuration discussed in a number of previous publications for the detection of a variety of chemical species 2 4. A schematic of the sensor is shown in Fig. 1. An LED source operating at $860 \mathrm{~nm}$ and modulated at $10 \mathrm{kHz}$ is used to inject about $25 \mu \mathrm{W}$ of power into the optical fiber. Multimode fiber with a $50 \mu \mathrm{m}$ diameter core and $125 \mu \mathrm{m}$ total diameter is used. Light passes through the 
coupler to the sensing fiber end. The fiber end is cleaved using a York model FK11 cleaver and the sensing film is evaporated in vacuum. Part of the light reflected from the fiber end passes through the coupler to the photodetector where it is synchronously detected. This basic configuration is suitable for use in the laboratory but not for practical use since there is no mechanism to calibrate the system for fiber and connector losses.

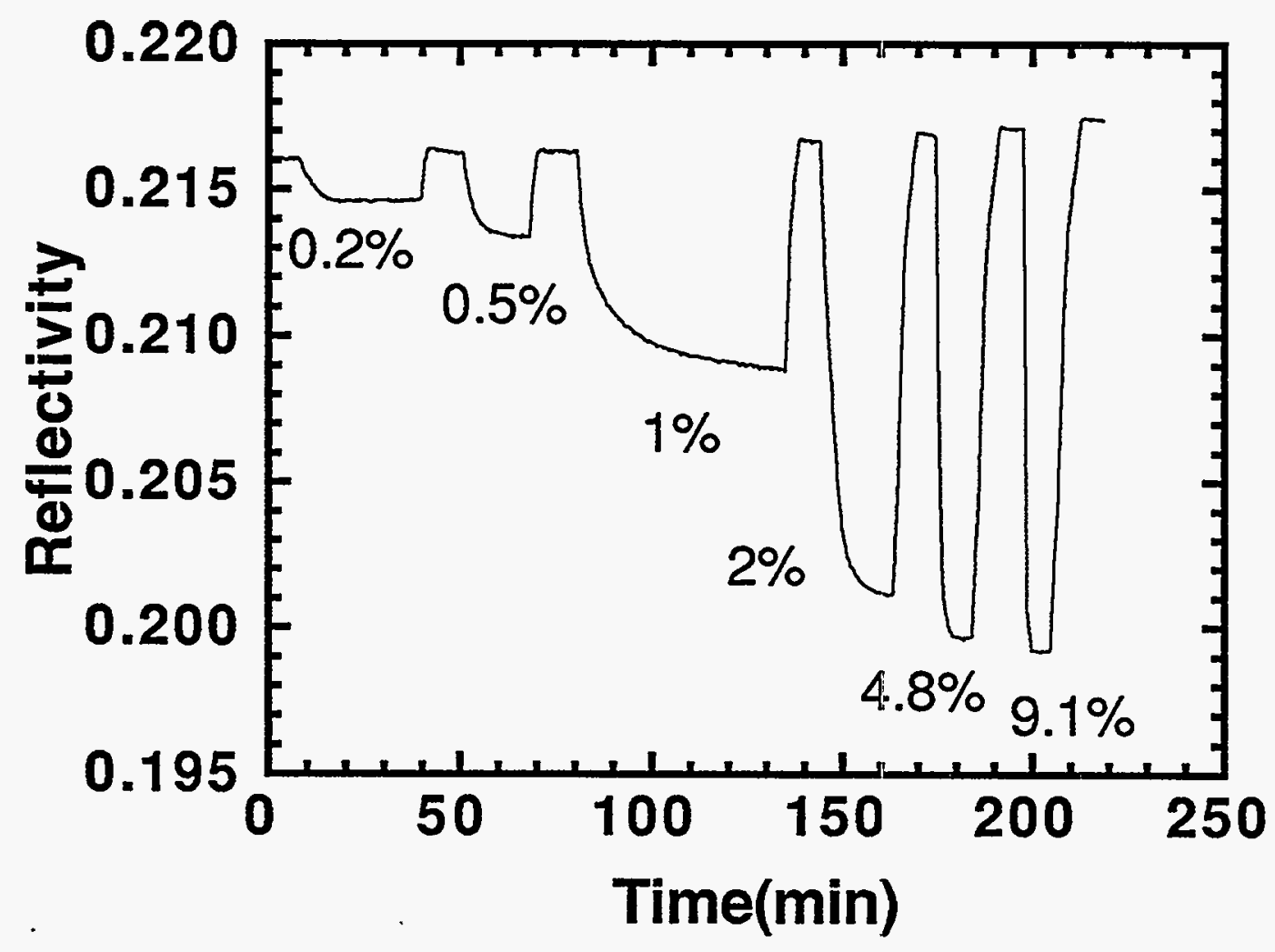

Fig. 2 Response of a $10 \mathrm{~nm}$ thick Pd film on the fiber end exposed to various concentrations of $\mathrm{H}_{2}$ in a $\mathrm{N}_{2}$ carrier gas. .The system is flushed with air between exposures.

The light penetrating the sensitive coating will go as far as the optical properties of the coating allow. Thus for metals the optical penetration depth is on the order of a tens of nanometers and for dielectric materials such as plastics the penetration depth can be many microns. The penetration depth of the light determines that part of the sensitive coating to which the sensor will respond. Thus for thin metal films the light will sense changes in the optical properties of the "bulk" metal as well as the outer surface. For optically thick metal films the light will only sense changes in the bulk optical properties. The interaction of the surface phase of a material can be quite different than from the bulk. Thus thin metal films automatically qualify as two phase sensing materials and effects due the chemisorption reactions as well as bulk chemical reactions should be observable. The response of an optically thin palladium film to various concentrations of $\mathrm{H}_{2}$ in $\mathrm{N}_{2}$ is shown in Fig. 2. Distinct effects from the bulk and surface are not obviously evident. The results do show a response that increases with $\mathrm{H}_{2}$ concentration and suggest a sensitivity capable of detecting hundreds of $\mathrm{ppm}$ in $\mathrm{N}_{2}$. Two limitations of using pure palladium as the sensing material is that the sensitivity is not good enough for the CRADA project which 
must detect $10 \mathrm{ppm}$ and below in oil and that the phase transition in the hydride at high concentrations can destroy the film. Both of these problems suggest examination of various palladium alloys as sensing materials to enhance sensitivity and improve durability.

Another goal of the project was the development of a prototype instrument that can be used in the field as well as compensate for environmental effects on the sensor response such as diumal temperature fluctuations. The final instrument we built for this project was a significant departure from any previous instrumentation in that the number of components was greatly reduced and a micro-processor was incorporated to control the measurements, provide an interface to the outside world, and provide the capability to incorporate calibration functions.

\section{Science of the Hydrogen Sensing Coatings}

The response of the hydrogen sensor seen in Fig. 2 arises from the interaction between hydrogen and the palladium to form a metal hydrogen alloy (hydride). The "hydride" has modified optical constants due to the changes in electronic structure relative to the pure metal, induced by the presence of the hydrogen. "Hydride" formation has been extensively studied5. Previous experiments ${ }^{2}$ have shown that the size of the response is proportional to the concentration of hydrogen in the "hydride". Thus the sensitivity to hydrogen is expected to be determined by the solubility of hydrogen in the sensing coating. This can be changed in palladium alloys by varying composition as well as the alloy components. Thus hydrogen is less soluble in PdNi alloys than in pure Pd and more soluble in PdAu and PdAg alloys. The later two have been explored in detail for this project.

The use of alloy coatings for sensing applications raises a number of issues that need to be addressed. The first is the method of deposition since an alloy cannot be evaporated and deposited with the same composition. Alternate methods of deposition are sputtering and evaporation using two sources, one for each alloy component. Sputtering was used initially because of the simplicity of the method. However, it is difficult to control the deposition conditions with the particular instrument we chose. The sputtering system chosen is manufactured by SPI Supplies Inc. It is a modified diode magnetron sputtering system that is connected to a vacuum control unit and is intended for coating of electron microscopy targets. The system is compact, easy to use, and was inexpensive to purchase. The alloy targets were also manufactured by SPI Supplies Inc. The sputter "magnetic head" design allows for very rapid coating. The penalty for having a very rapid coating system is that the alloy particles are very large compared to the particle size of an E-beam evaporation deposition, thus a more porous film is put down onto the substrate. In addition, the vacuum system the sputtering system used could only reach a base pressure of $2 \times 10^{-2}$ Torr. At these pressures impurities in the chamber, such as water and oxygen, were deposited onto the substrate along with the alloys. The oxygen resulted in growth of silver oxide throughout the film. This problem was compounded when it was necessary to break vacuum between metal coatings to change sputtering targets. A partial solution to the contamination problem was achieved by flushing the chamber at least 3 times with UHP Argon gas while pumping it down to base pressure between each flush cycle. While films grown with this procedure were much improved, they still did not achieved the expected performance in a consistent manner. Best results using DC sputtering deposition was achieved using Argon as the plasma gas and a current of $30 \mathrm{~mA}$.

Subsequently, a change was made to using dual e-beam deposition (Angstrom Sciences) with multiple targets to eliminate the need to open the vacuum chamber between metal coatings. This greatly improved the reproducibility of the films and the overall performance. The final sensor configuration used this deposition method for both the hydrogen sensing films and the temperature sensing films.

A second issue raised by the use of alloy coatings is the stability of the coatings. Because of the very small dimensions of the films (tens of $\mathrm{nm}$ thick) diffusion and surface segregation can be of concern. Initial measurements were made with $\mathrm{PdAu}$ alloys that confirmed the expected enhanced response at low $\mathrm{H}_{2}$ concentrations due to increased solubility of the hydrogen in the alloy 6 . However, stability of the film was a problem. Apparently Au atoms can move easily in the 
film and produce changes in the speed of response of the sensor. It is speculated that the Au segregates at the surface resulting in the slow down in sensor response. Palladium-silver also offers enhanced solubility of hydrogen at low concentrations ${ }^{7}$ and so the experimental effort was focused here. Again there was evidence of a slowing down in the response of the sensor over time attributed to the segregation of silver to the surface and subsecquent growth of an oxide at the surface. Other evidence is provided by the fact that the optical response of the sensor for such thin films will be to both changes in the "bulk" and surface of the film. This is clearly seen in Fig. 3 for a thin PdAg alloy. Exposure of PdAg to air produces a thin oxide layer that reduces the

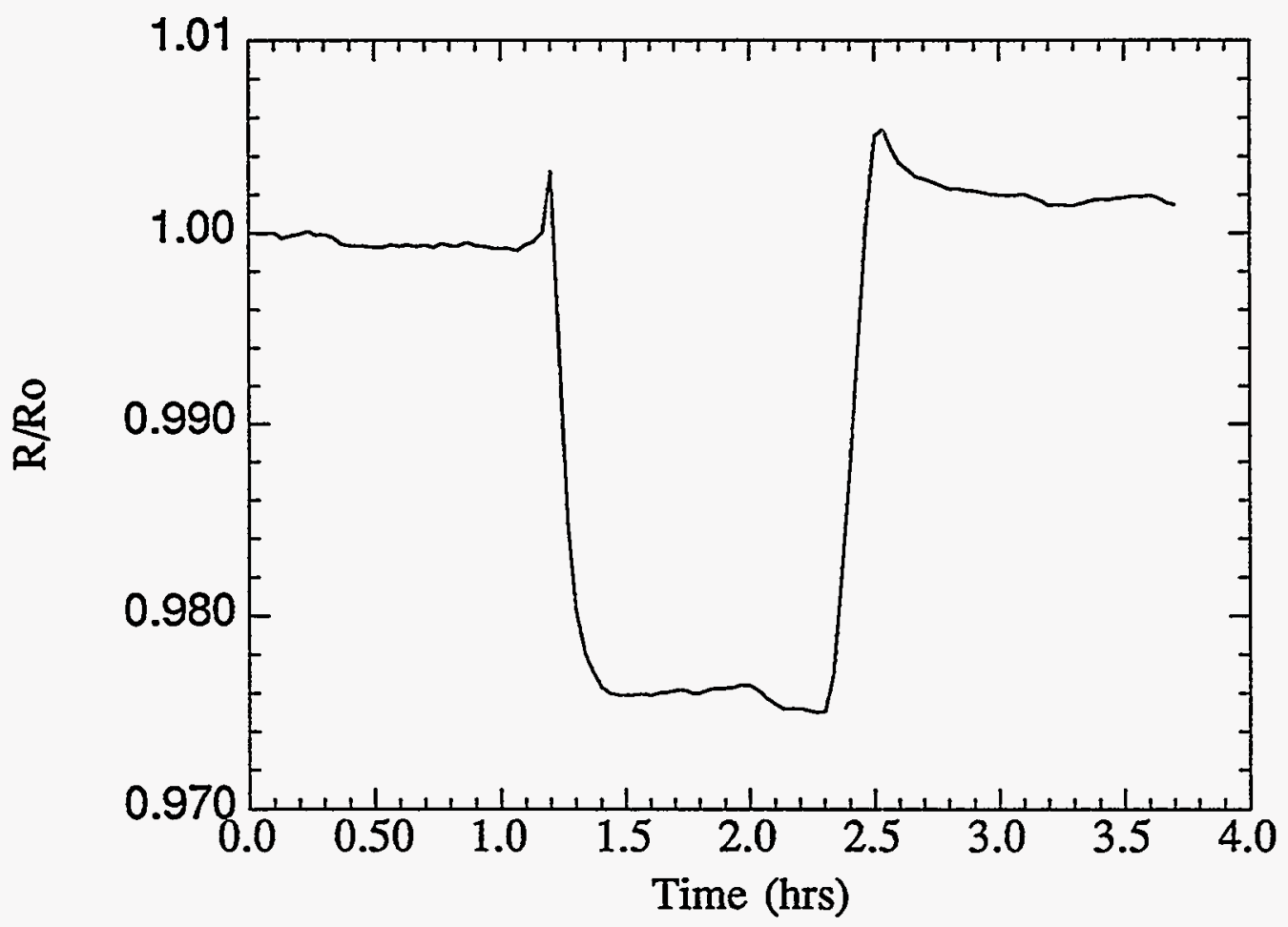

Fig. 3 Reflectivity of a $10 \mathrm{~nm}$ thick PdAg alloy film exposed to air, hydrogen, and then air. The spikes indicate the effects due to oxide growth on the alloy surface when exposed to air.

reflectivity for this optically thin film. When this film is then exposed to $\mathrm{H}_{2}$, the initial effect is the reduction of the oxide to metal which increases the reflectivity followed by a reduction in reflectivity as the bulk PdAg is converted to the hydride. Subsequently, the exposure of the sensor to air then produces an increase in reflectivity as the hydride is returned to metallic Pd and a decrease as the oxide layer is regrown. Such changes in the response of the sensitive layer implies the need for a more complex sensing coating structure that can respond to the presence of $\mathrm{H}_{2}$ and control the mobility of the alloy components. Furthermore, the use of an optically thick sensing coating will simplify the sensor response by limiting it to changes in the "bulk" alloy material. Since hydrogen has very high mobility in palladium alloys and the films are very thin, an optically thick layer will not inhibit the sensor response time.

A third concern with any thin metallic coating as the sensing layer is the effects of stress on the thermodynamics of hydrogen uptake. This is illustrated for thin films of pure palladium in Fig. 
4. This shows the equilibrium reflectivity change for a thin film with and without an underlayer of nickel. The argument here is that the nickel acts as an adhesive layer making a good bond between the fiber end and the palladium film. The resulting restriction on lateral movement, that does not appear to occur for palladium films without the nickel, induces stress in the film when hydrogen atoms are inserted 2 . This additional mechanical energy term changes the thermodynamics as indicated by the different response of the two films for the same concentration of hydrogen. This differentiation occurs primarily at high hydrogen concentrations where the mechanical energy contribution is greatest. It is less important at the lower concentrations of interest here. It is important however to provide an adhesive layer for reproducibility of the response and ruggedness of the sensing coating.

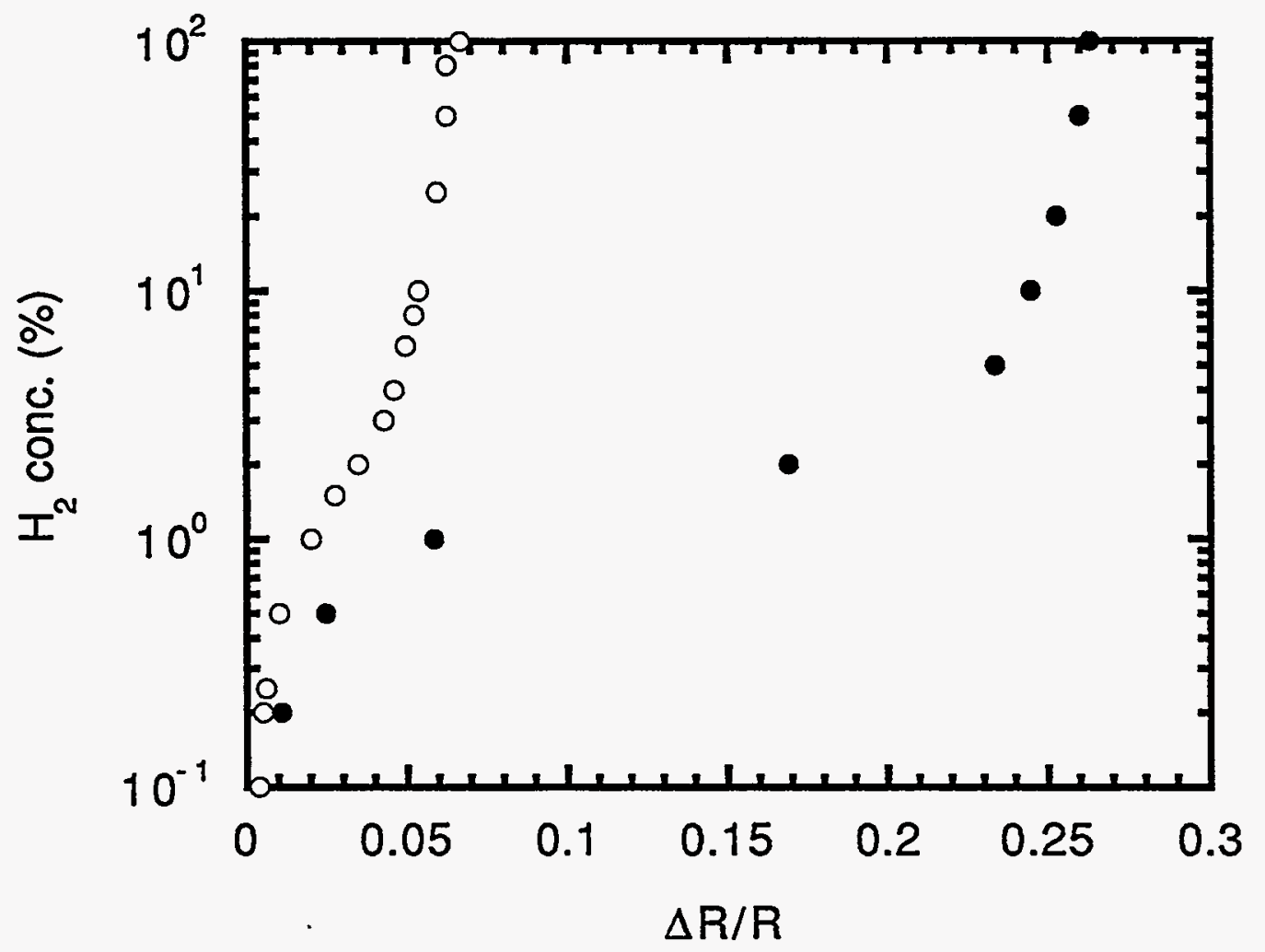

Fig. 4 Normalized reflectivity change versus hydrogen concentration in $\mathrm{N}_{2}$ for $10 \mathrm{~nm}$ films of pure palladium with (open circles) and without (filled circles) a thin underlayer of nickel.

While the introduction of a multi-layered sensing coating can alleviate some of the limitations associated with simpler coatings, it can also lead to unusual and unexpected phenomena in the sensor response. The kinetics of the hydrogen uptake process depend on the surface reaction and the diffusion of the species through the film. However, interesting effects on the kinetics can result when a composite layered film structure is used. Consider a hydrogen sensor that uses a 2 layer sensing film of a palladium alloy and pure palladium. The alloy, a PdAg alloy, is used to increase the solubility and thus the optical response of the sensor for a given partial pressure of hydrogen. The pure Pd layer which is the outer layer is used to limit effects of oxygen on the response of the sensor. This composite structure produces a sensitive response without the oxidation of the surface that would slow the kinetics. 
The alloy layer is thicker than the light penetration depth into the sensing layer. Thus the optical response is from the changes in the alloy properties only. Formation of the hydride with exposure to hydrogen results in a reduction in reflectivity. Hydrogen can easily diffuse from the pure Pd layer into the alloy layer. The pure Pd layer stops oxidation of the silver alloy and formation of AgO on the surface of the sensor. Such a layer impedes the transport of hydrogen into the sensing layer and slows the response of the sensor. Response of this sensor for a range of concentrations of hydrogen is shown in Fig. 5. An unusual feature of this data is the step in the

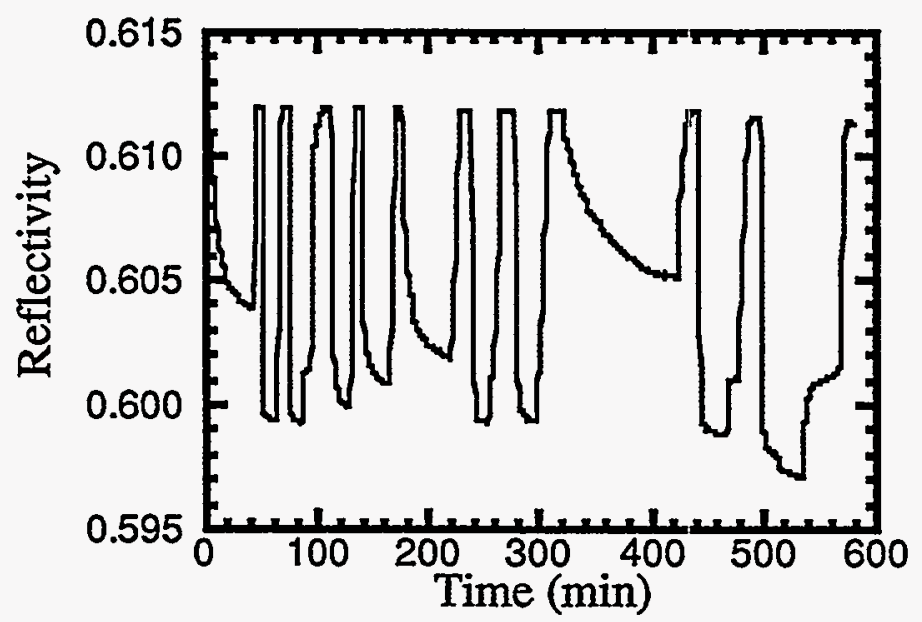

Fig. 5. Response of 2 layer hydrogen sensor to repeated hydrogen pulses at the following partial pressures of $\mathrm{H}_{2}$ in $\mathrm{N}_{2}$ in Torr; $1.24,21.7,21.7,12.4,6.2,3.1,18.6,15.5,0.62,31.0$, and 62.0 altemated with dry air.

recovery of the sensor when exposed to dry air. This step becomes more evident as the hydrogen concentration is increased above 15 Torr. Such a step is not evident with the uptake of hydrogen. If this were characteristic of the thermodynamics of the sensor and was an equilibrium phenomena, it should occur on hydrogen uptake as well as removal and should be evident when the peak response is plotted versus hydrogen concentration. Neither is true. Thus this anomalous response must be related to the kinetic processes associated with removal of hydrogen from the sensor. Since a similar behavior is not observed in either pure Pd or its alloys alone, it is tempting to attribute it to the composite nature of the sensing film. The explanation of this phenomena can be found by examining the adsorption isotherms shown in Fig. 6.

Consider now the filling of this composite structure with hydrogen when the hydrogen partial pressure is raised to some fixed value such as 62 Torr. Assuming equilibrium between the pure $P d$ and the alloy, the hydrogen will initially go almost exclusively into the alloy since the solubility is much larger than in pure $\mathrm{Pd}$. As the $\mathrm{H} / \mathrm{M}$ ratio in the alloy approaches about 0.15 , more and more hydrogen will go into the pure Pd as indicated by the isotherm until the H/M ratio in pure Pd approaches 0.6. After this point hydrogen will go in similar amounts into both the pure $\mathrm{Pd}$ and Pd alloy. Assuming equilibrium between the pure metal and its alloy means that at a given point in time the relative hydride compositions of the two layers can be determined from drawing a horizontal line on Figure 6. Similarly the removal of hydrogen from the composite film will follow in a similar fashion as long as the equilibrium between the pure Pd and alloy is maintained. Since the sensor only responds to the hydride composition of the alloy the dynamics of the response can 
be distorted by the equilibrium between the two layers. The peak response of the sensor will of course follow the alloy isotherm and will not be expected to show a plateau region. For hydrogen

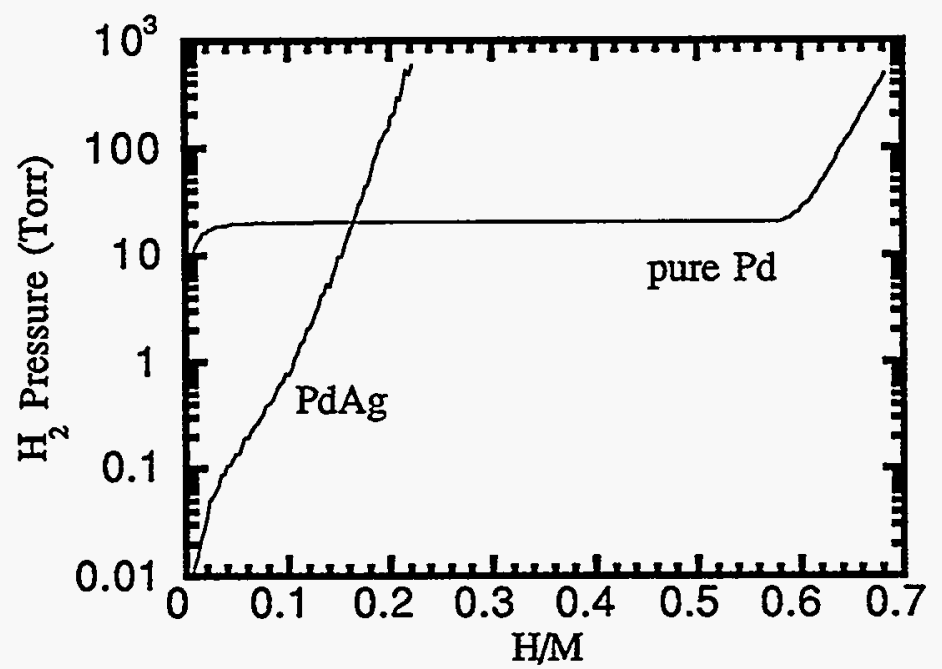

Fig. 6. Adsorption isotherms for bulk samples of pure Pd and PdAg alloy at room temperature. $\mathrm{H} / \mathrm{M}$ is the hydrogen to metal atom ratio in the hydride.

uptake the surface reactions are rapid as indicated by the sensor response and the plateau region is not clearly evident. It is only for removal of hydrogen by catalytic burning that the plateau region is so evident due to the slow reaction rates at the surface of the sensing film. This kind of kinetic sensor response should be observable whenever a composite film structure is used for sensing purposes, the two or more layers have different adsorption isotherms, and the sensor responds to the uptake of chemical species in only one of the layers.

A final consideration for these sensor coatings is the response to other gases. In a large power transformers the sensor can be expected to be exposed to varying concentrations of a number of gases and it is important to know if such gases can affect the performance of the sensor. Gases of concern include: $\mathrm{N}_{2}, \mathrm{O}_{2}, \mathrm{H}_{2} \mathrm{O}, \mathrm{CO}, \mathrm{CO}_{2}$, and various small molecule hydrocarbons. Tests showed that only $\mathrm{O}_{2}$ influenced the response of the sensor in a significant manner and then only when the concentration of $\mathrm{O}_{2}$ was an order of magnitude larger than the $\mathrm{H}_{2}$. This is illustrated in Fig. 7 which shows the response of a sensor to the presence of oxygen at low partial pressures of hydrogen gas. In addition to reducing the size of the response the presence of oxygen also slows the rate of response of the sensor. The first phenomena comes from the catalytic burning of hydrogen on the surface of the sensor to produce water. This reduces the steady state concentration of hydrogen on the surface of the coating and thus in the "bulk" since they are in thermodynamic equilibrium. The slower response probably arises from the oxygen occupying sites on the surface of the coating and limiting the access of the hydrogen. The resulting limitation of available reaction sites constrains the rate at which hydrogen can access the interior of the coating and thus the sensor response. These results show that when the oxygen concentration reaches ten times the hydrogen concentration, significant changes in sensor response can occur. This fact needs to be kept in mind in all sensor applications. It should be pointed out that this effect is intrinsic to the chemistry of palladium containing alloys and not unique to this fiber optic sensor. It applies to any chemical sensor using these sensing materials.

The fiber optic configuration for chemical sensing provides a platform for the sensing application. It is the gas/solid interfacial chemistry of the sensing coatings which determine overall sensor performance and introduces limitations to the sensor performance. These results show that hydrogen can be detected successfully at the desired concentrations for power transformer 
applications using palladium alloys. In addition, several limitations have been identified. Oxygen concentrations ten times larger than the hydrogen concentration will affect sensor performance. Use

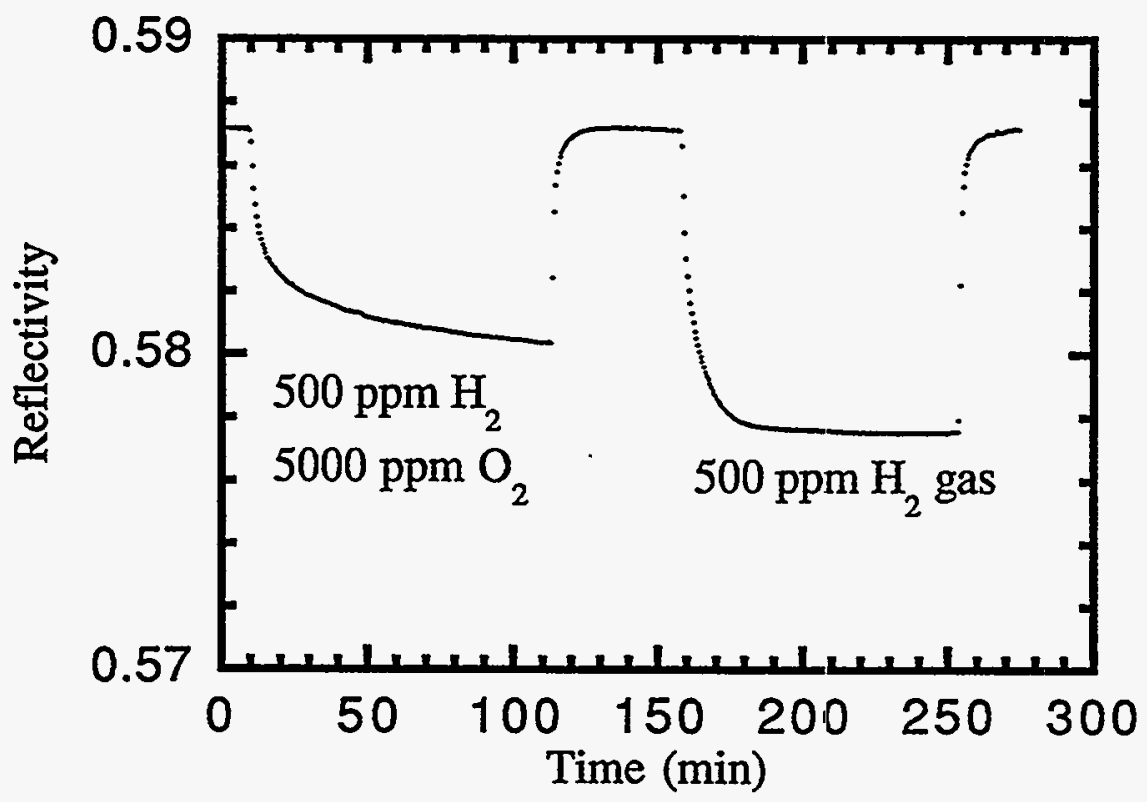

Fig. 7 Response of $\mathrm{PdAg}$ alloy sensor to $500 \mathrm{ppm} \mathrm{H}_{2}$ in $\mathrm{N}_{2}$ with and without the presence of $\mathrm{O}_{2}$. Between these exposures the sensor is exposed to dry air.

of alloys in these films with very small dimensions means that diffusion of one alloy component, silver or gold, can cause instabilities in the film and degrade sensor performance. This problem is also tied in with the film adhesion and stress induced in the film from its multi-layer structure. An ideal film for this sensor application has not yet been defined. Because the sensor response is proportional to the concentration of hydrogen in the sensing coating, which is the result of an activated chemical reaction, the response will be temperature dependent. For this reason a temperature measurement at the hydrogen sensor is required to properly interpret the sensor response. This problem is addressed in the next section.

\section{Temperature Sensing}

Fiber optic temperature sensing is one of the most developed applications for fiber optic sensors with at least two instruments commercially available in this country (Photonetics and Luxtron). Detailed descriptions of the mechanisms of operation can be found in reference 8 . The reason that commercial products were so quickly developed compared to other types of sensor is the existence of a market with unique needs. Food processing with microwave ovens requires a non-metallic temperature sensor for control and processing development. For our application one of these commercially available instruments could of course be used. However, this would result in additional instrumentation and cost. Thus we chose to develop a fiber optic temperature sensor that utilized the same electronics package as the hydrogen sensor to limit additional cost and to improve compatibility in our application.

With the two wavelength $(810 \mathrm{~nm} \& 880 \mathrm{~nm}$ ) sensing approach taken with the hydrogen sensor (see section IV.) it seemed appropriate to follow previous efforts $9-11$ and build a temperature sensor which utilizes a thin silicon film on the end of the fiber. This film forms a 
small interferometer that changes its reflectivity with temperature due to the temperature dependence of the refractive index of the silicon. By using two wavelengths and ratioing the resulting signals, effects of other environmental factors are eliminated. The silicon was deposited on the cleaved end of a 50/125 $\mu \mathrm{m}$ fiber by e-beam evaporation. The reflectivity of the end was

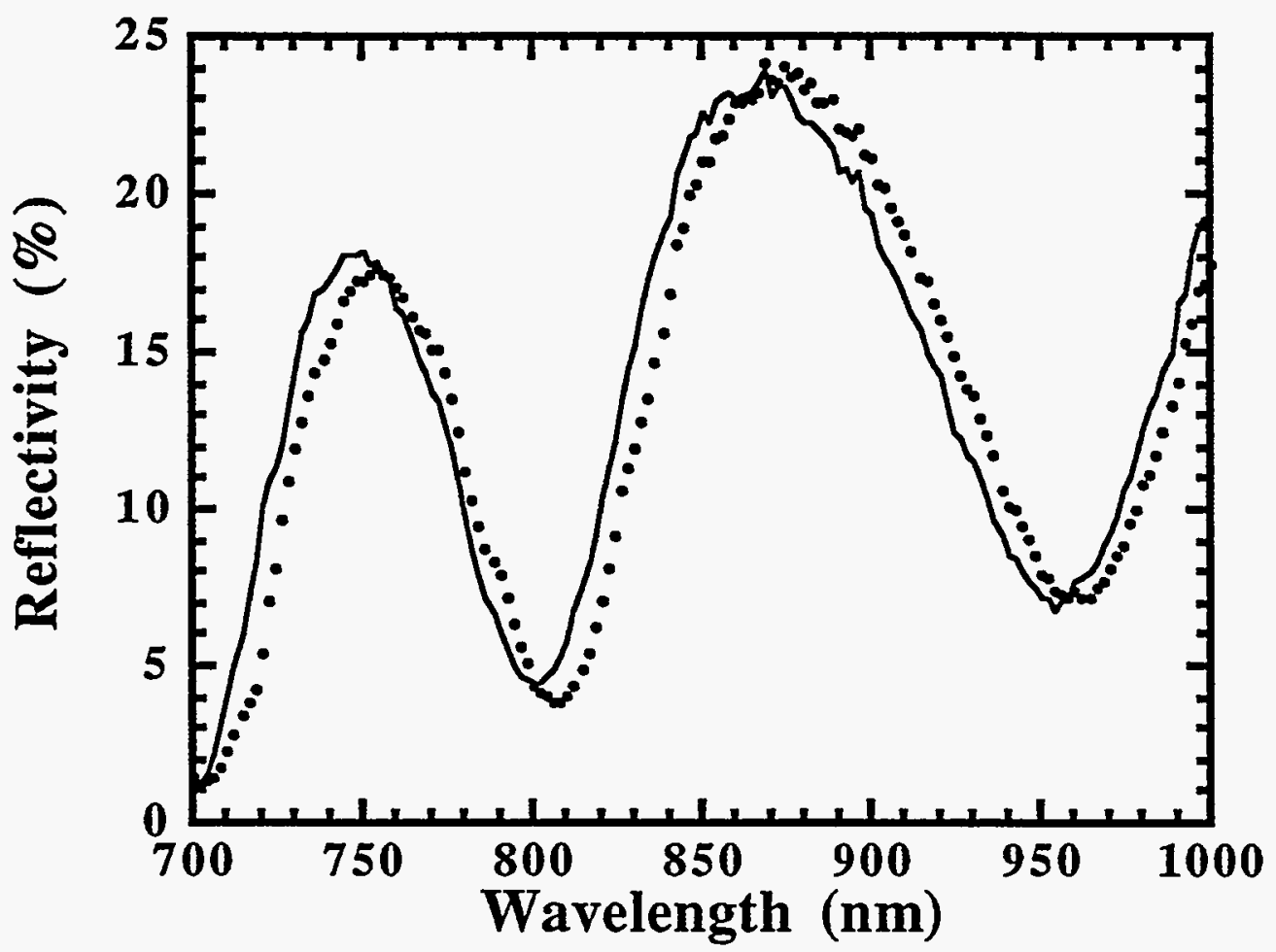

Fig. 8 Reflectivity of a silicon temperature sensor coating at $0^{\circ} \mathrm{C}$ (solid line) and $80^{\circ} \mathrm{C}$ (dots).

monitored during deposition to determine the optimum time to terminate the deposition. Approximately $500 \mathrm{~nm}$ of silicon is deposited followed by $100 \mathrm{~nm}$ of nickel. The nickel protects the silicon from corrosion and provides an optical seal so that the light does not experience the environment in which the sensor operates. Reflectivity changes due to temperature were optimized at the two wavelengths to give an increase at one wavelength and a decrease at the other. This is illustrated in Fig. 8 which shows increasing reflectivity with increasing temperature at $880 \mathrm{~nm}$ and decreasing reflectivity with increasing temperature at $810 \mathrm{~nm}$. Thus the overall response is a result of the response at both wavelengths. Using this sensor and the electronics package developed for sensing hydrogen, temperature can be measured with an accuracy of about one half degree centigrade. This is illustrated in Fig. 9 which shows a comparison between the fiber optic temperature sensor and a thermocouple. The two sets of data are offset by 3 degrees to facilitate the comparison. This accuracy is good enough to enable correction of the hydrogen sensor data for temperature variations. Temperature is also a useful parameter to know in large power transformers in its own right. Local heating can result from corona or eddy current losses. 


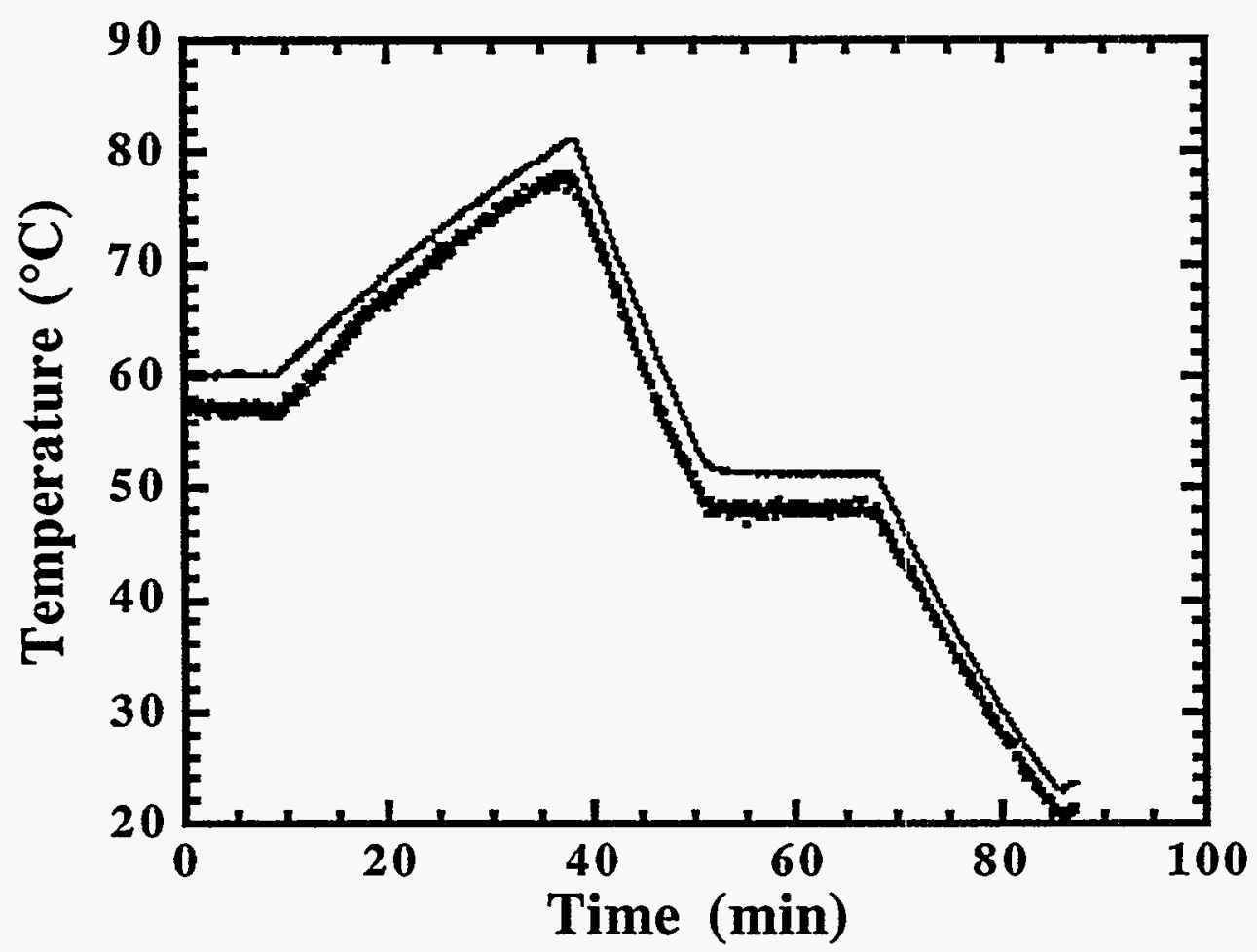

Fig. 9 Comparison between the fiber optic temperature sensor (dots) and a thermocouple (line).

\section{Engineering the Sensing Elements}

Initially, 50/125 $\mu \mathrm{m}$ multi-mode fiber with an appropriate coating on the cleaved end was used for hydrogen and temperature sensing. As the coatings became multi-layered and a two wavelength system was adopted it became less and less practical to use the fiber tips. For a practical device some consideration must be given to the engineering aspects of manufacture. It is for this reason that quarter pitch GRaded INdex lenses (NSG Glass) were adopted as substrates to hold the hydrogen sensing coatings. With the two wavelength system a selective filter must be applied that reflects the longer wavelength and passes the shoiter wavelength. This allows the shorter wavelength to sample the sensing coating while the longer wavelength does not. The ratio of these two signals responds to hydrogen but also corrects for any time varying optical losses in the system. Since stresses generated by the sensing coatings when deposited directly on the high pass filters caused pealing, it was necessary to deposit them on opposite ends of the GRIN lens as shown in Fig. 10. These wavelength selective coatings were deposited by CVI Laser Inc. The 


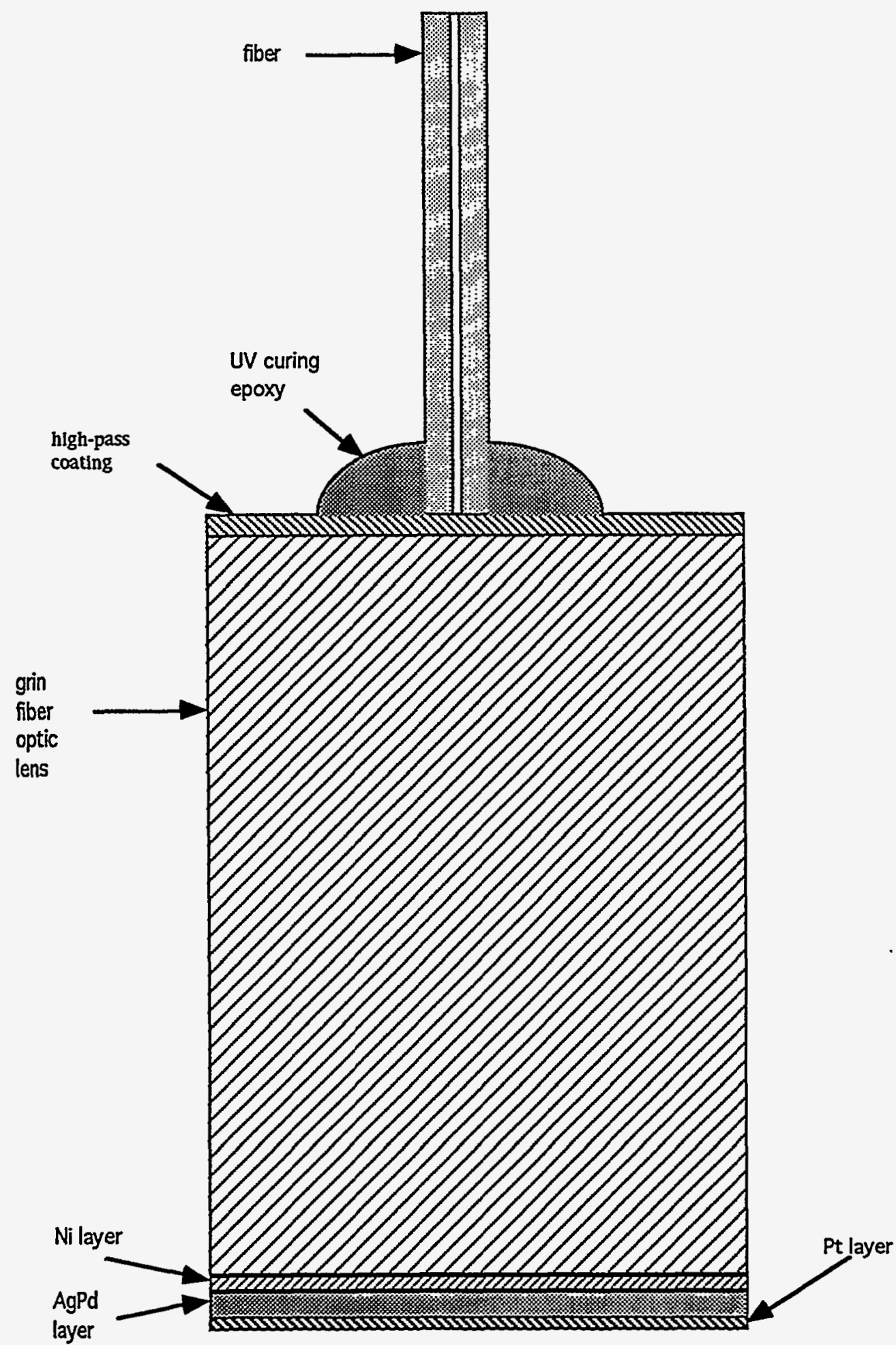

Fig. 10 Configuration of the hydrogen sensor using GRIN lenses to hold the sensing coatings and the wavelength selective filter.

spectral response of a typical coating on a GRIN lens is shown in Fig. 11. Thus the $880 \mathrm{~nm}$ light is reflected and does not change intensity with hydrogen exposure while the $810 \mathrm{~nm}$ light does 
respond to hydrogen exposure. The multi-layer sensing coating has an underlayer of $10 \mathrm{~nm}$ of nickel which is an adhesive layer yet passes most of the light. Next comes an optically thick layer, $150 \mathrm{~nm}$ of PdAg, the sensing layer followed by $25 \mathrm{~nm}$ of Pt which protects the Ag from oxidation and still allows passage of the hydrogen. Unfortunately the Pt layer is not completely impervious

High Pass Filter

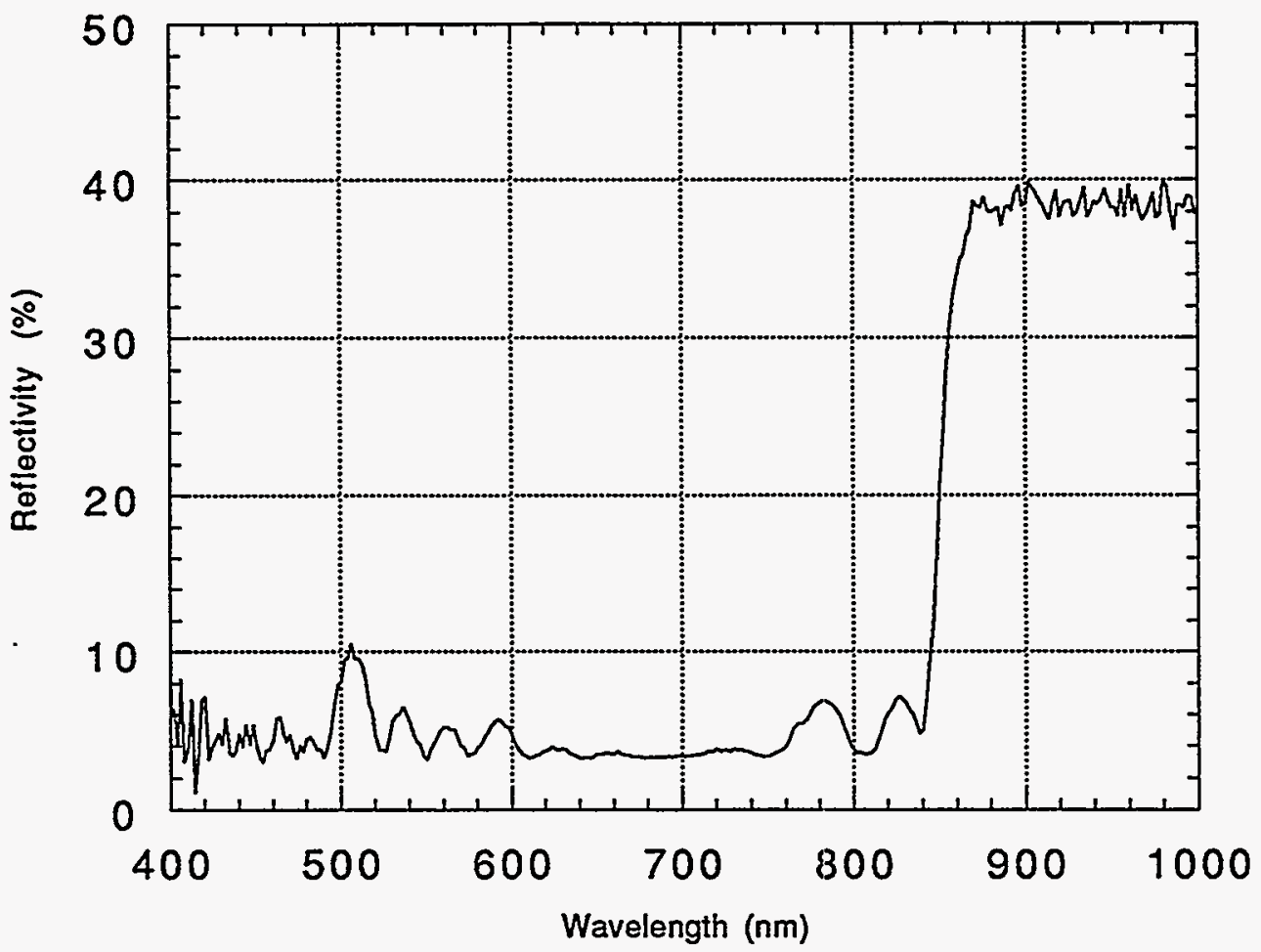

Fig. 11 Reflectivity spectrum of wavelength selective filter applied to GRIN lens.

to Ag migration and so the sensor degrades its response by getting slower over time. This is shown in Fig. 12; sputter Auger profiles of the various metals throughout the multi-layer structure. Within the resolution of these measurements there appears to be little intermixing of the layers. However, upon close examination there is a hint of enhanced silver concentrated at the surface. Further evidence of $\mathrm{Ag}$ migration comes from measurement of the time it takes for the sensor response to slow down as a function of Pt layer thickness. These results show a quadratic dependence on thickness as one might expect for some species diffusing through the Pt layer. Thicker Pt layers which would improve sensor lifetime also increase stresses in the coating and cause mechanical failure. A suitable diffusion barrier for silver has not yet been identified. This multi-layer structure is the best configuration yet identified. It is clear however that further development of the sensing layer is necessary for successful commercialization of this device. Attachment of the fiber to the GRIN lens is done using active alignment in which the reflectivity from the fiber is monitored while the position of the fiber on the GRIN lens is adjusted. For these quarter pitch lenses reflectivity is a maximum when the fiber end is exactly in the center. After this point is located UV curing epoxy is applied and cured. Subsequent to this the lens/fiber assembly must be packaged in such a way as to provide protection for the sensor while allowing it to be exposed to the environment to be monitored. In the present application the probe must withstand temperatures up to $125^{\circ} \mathrm{C}$ and exposure to transformer oil. A simple packaging method 


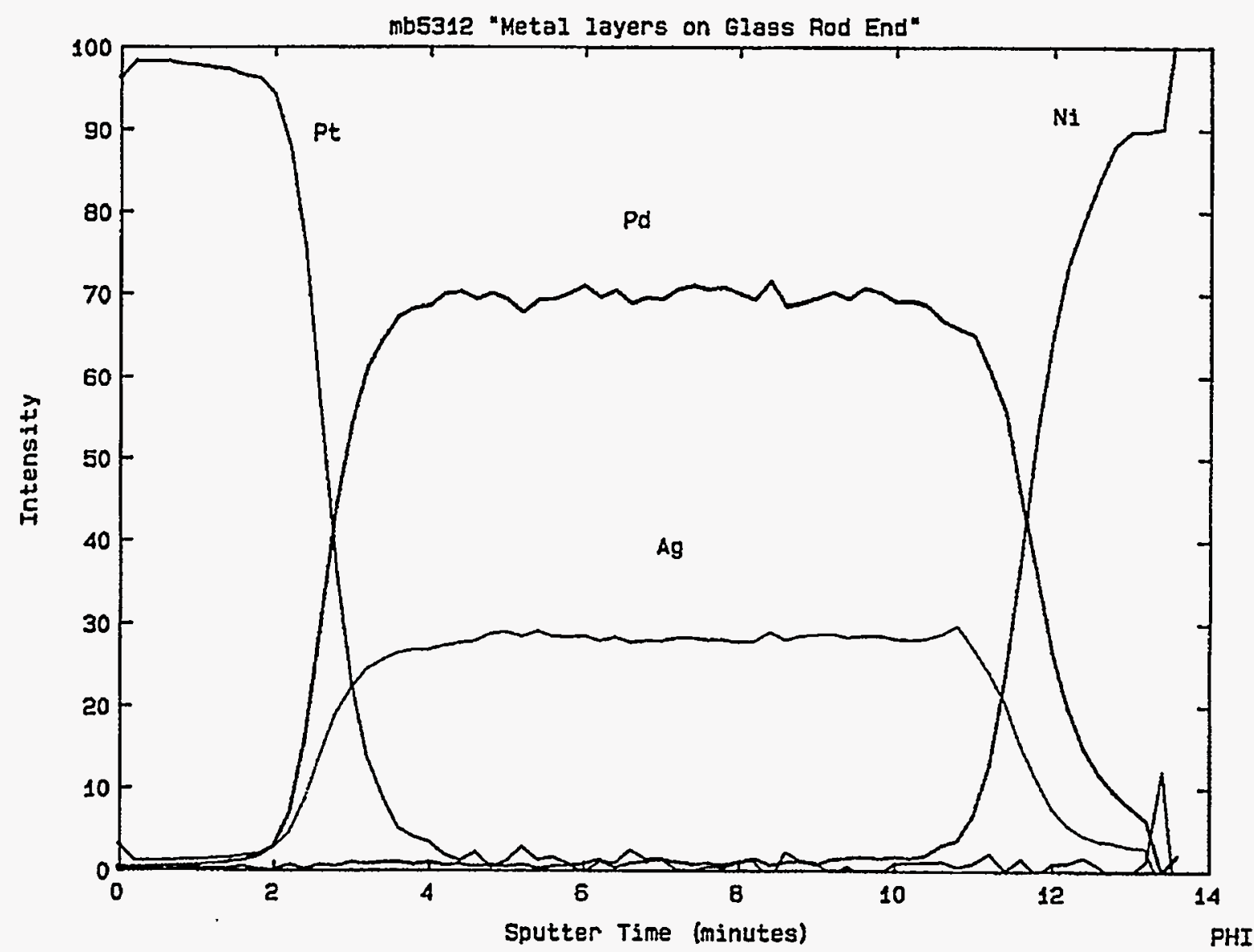

Fig. 12 Sputter Auger profile for the multi-layer sensing coating on the end of a GRIN lens. The sputter rate was approximately $175 \AA / \mathrm{min}$. on a standard $\mathrm{SiO}_{2}$ specimen.

suitable for prototyping applications is shown in Fig. 13. First the fiber attached to the lens is cleaved and fusion bonded to a longer jacketed fiber. This assembly is then epoxied into a ceramic tube with the epoxy serving as a bonding agent and filler. This configuration provides a mechanically rugged assembly with strong attachment to the jacketed fiber.

For the temperature sensor the wavelength selectivity is intrinsic to the silicon coating and thus the coating will consist of only two layer (Si/Ni). For this limited complexity the decision was made to continue coating the fiber tips rather than introduce the GRIN lenses. Furthermore, the temperature sensor does not need to be directly exposed to the transformer oil as does the hydrogen sensor. Thus it was initially decided to completely encase it in epoxy within a ceramic tube. With this configuration there were a number of failures in which it appeared that the sensing film was pulled away from the fiber end. A tentative explanation of the problem is differential thermal expansion between the epoxy and the coated fiber end. An alternated packaging configuration designed to alleviate this problem in which the fiber is enclosed in a sealed glass tube is shown in Fig. 14. So far this design has been successful with no failures. It does not appear that vibration of the free fiber end is a significant problem. Without further knowledge of the specific application of the sensors, a more specific package is not possible. The packages developed offer all the characteristics desired for the transformer application. It may be desirable to modify the packaging to simplify manufacturing of such devices. Field experience is necessary to determine ultimate feasibility of using these designs. 


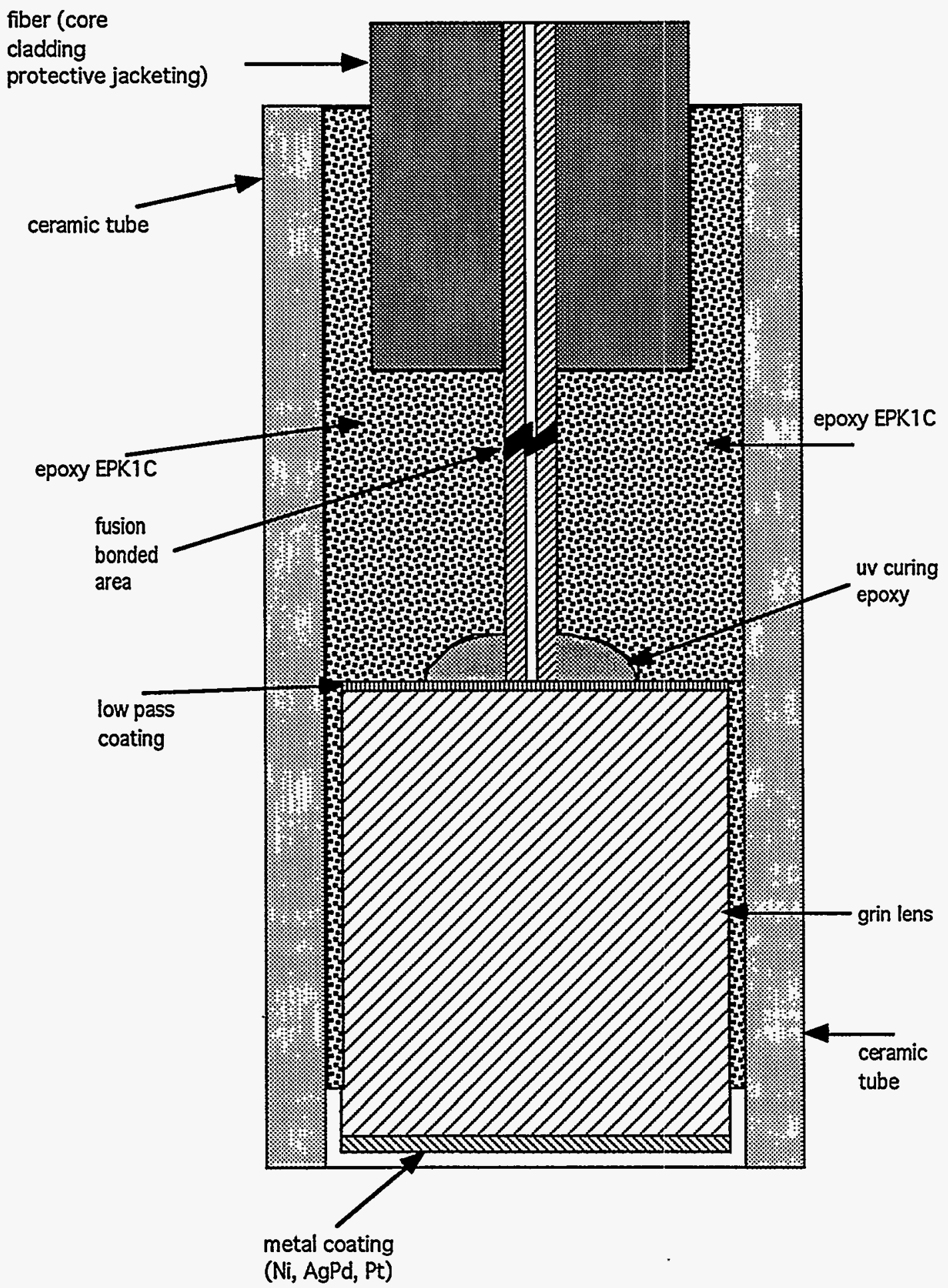

Fig. 13 Packaging of the prototype hydrogen sensor for use in transformer oil. 


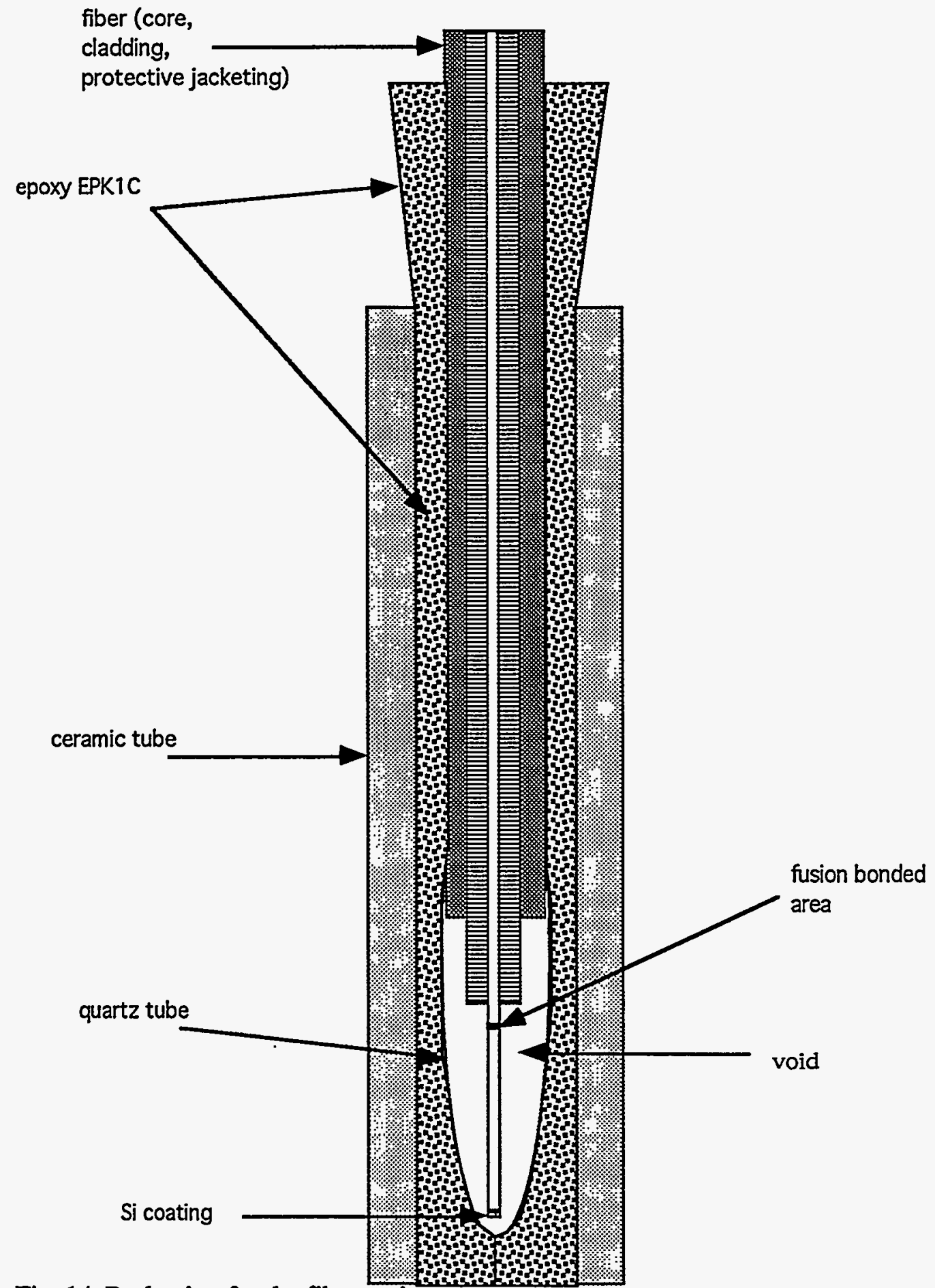

Fig. 14 Packaging for the fiber optic temperature sensor.

\section{Instrument Design and Development}

With an acceptable design for the sensing elements the overall prototype instrument design can be considered to achieve a device that is self operating, portable, fieldable, and cost effective. The fundamental instrument requirements include: (1) the use of two wavelengths to enable 
corrections for inadvertent optical losses, (2) measurement of both $\mathrm{H}_{2}$ and temperature to allow correction of the hydrogen measurements for temperature effects, (3) microprocessor control with built-in calibration functions, (4) RS-232 port to download calibration parameters, and (5) the ability to operate in auto and command modes and provide properly calibrated and corrected outputs of hydrogen concentration and temperature. The instrument also includes a liquid crystal

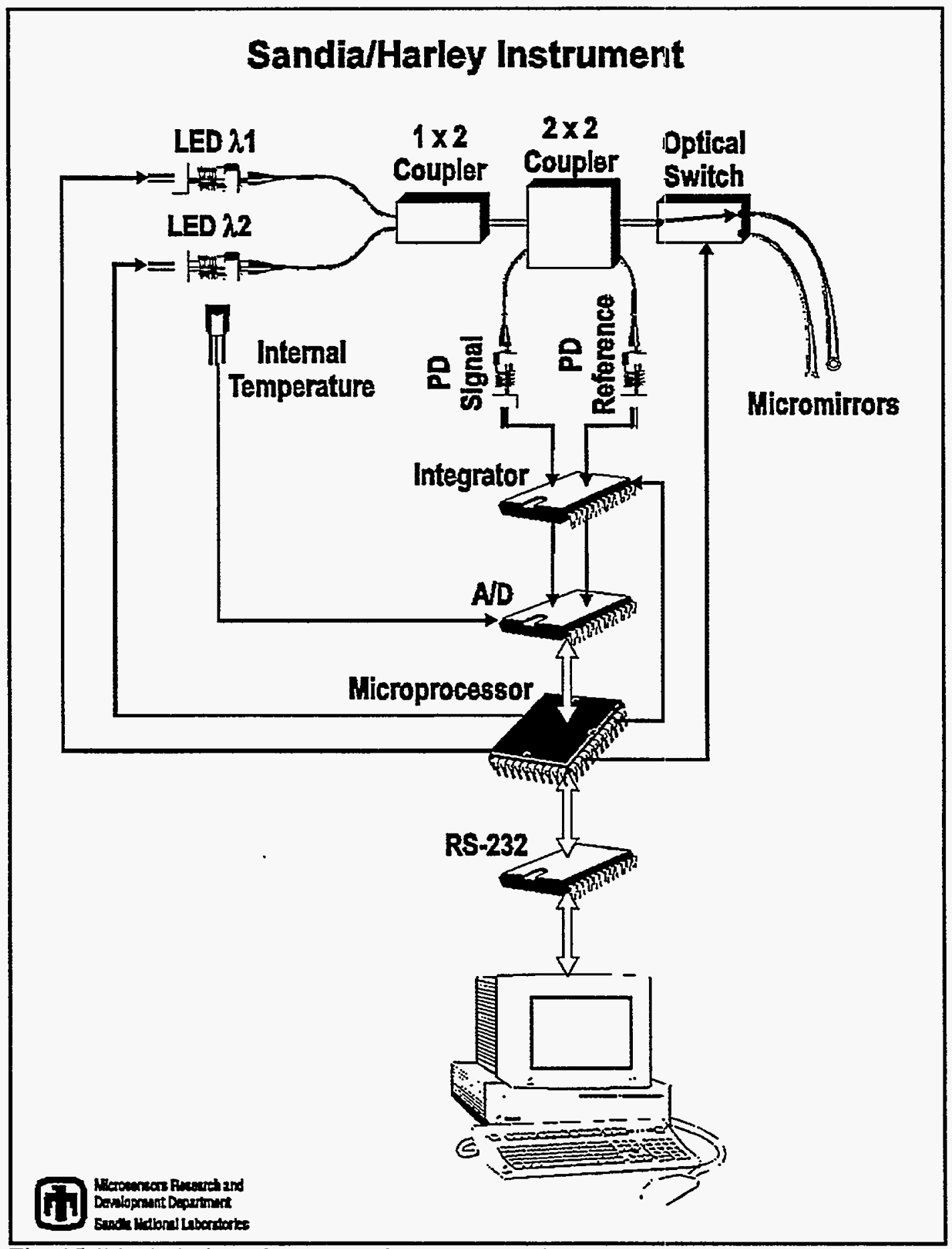

Fig. 15 Block design of prototype instrument package. 
display that reads out hydrogen concentrations in ppm or temperature in ${ }^{\circ} \mathrm{C}$ and two $4-20 \mathrm{~mA}$ current outputs for the same purpose. The basic overall design is shown in Fig. 15. The microprocessor provides properly timed and conditioned pulses to two LED drivers which drive LEDs at $810 \mathrm{~nm}$ and $880 \mathrm{~nm}$. These devices are tumed on sequentially to produce two optical pulses. The pulses are combined by the $1 \mathrm{X} 2$ coupler and put into a $2 \times 2$ coupler. Half of the light coming out of the $2 \times 2$ coupler is directed to an optical switch under microprocessor control used to choose the sensor to be interrogated. The other half of the light goes to a photodiode which is the reference measurement of light intensity. The reflected light from the sensor passes through the $2 \times 2$ coupler to a photodiode used to measure the signal. Both photodiodes are attached to integrators that accumulate the total charge optically generated in the photodiodes. The microprocessor knows which wavelength it is looking at because of the time division multiplexing. On an appropriate signal from the microprocessor the outputs of the integrators and an internal temperature measuring device are digitized and transmitted to the microprocessor. It uses these numbers to calculate the hydrogen concentration and temperature. Details of the software used to control the microprocessor are discussed below. The circuits are split into a digital and analog boards. The microprocessor and all output drivers comprise the digital board with the rest of the circuits comprising the analog board. The LED's, photo-diodes optical couplers, and optical switch are on a separate optical board. The optical board was designed to use ' $n$ ' sensors while still using the same electronics boards. In our case we are only using 2 sensors, but by installing a $1 \mathrm{x} \mathrm{n}$ optical switch and modifying the software slightly, any number of sensors could be used.

The schematic for the digital board is shown in Fig. 16. The sixteen lines to the microprocessor provide the method for instrument control. Through them the microprocessor controls:

1) The LED drivers, sending them positive pulses varying in length from $1-500 \mathrm{msec}$. (user selectable) led1 and led2 turn on the LED's for the specified amount of time.

2) The A/D converter: A0, A1, CS, CONV, CAL, SCLK are the 6 input control lines. Details of operation will be described under the analog circuit board discussion.

SDATA and DRDY are the 2 lines use to output data to the microprocessor.

3) The Integrator has 2 control lines. The HOLD and the RESET. The HOLD controls the connection of the signal from the photo-diodes into the integrator. It is normally high and goes low to begin operation. The RESET line resets the integrator. It is normally low and goes thigh to reset.

4) The Optical Switch. Two lines are controlled. B0 and B1. These lines are normally held low. When a high pulse is seen the switch will move to the channel selected, 1 or 2 , thus changing which sensor is being measured.

All of these lines are connected to the analog board by a ribbon cable. The schematic for the analog board is shown in Fig. 17. The timing sequence for the measurements is set by the led pulses, hold and reset commands, and the convert signal to the A/D converter. A typical timing sequence is shown in Fig. 18.

The basic timing sequence is set by the hold and reset signal to the integrator chip. When the hold is low the photodiode is connected to the integrator and charge is accumulated. Just before this process the integrator is reset to zero accumulated charge. The LED is turned on during the time the photodiode is connected to the integrator so that the photocurrent is accumulated. After this time period and before the next reset the convert line to the A/D converter is raised high and the output of the integrator is converted to a digital number. All conversion is done before the next reset and then the cycle repeats. Channels A and B are the signal and reference channels respectively. This timing sequence is for one LED measurement and must be repeated for the second LED and for dark measurements. In the later case the measurement sequence is run but there is no LED pulse to turn it on. 


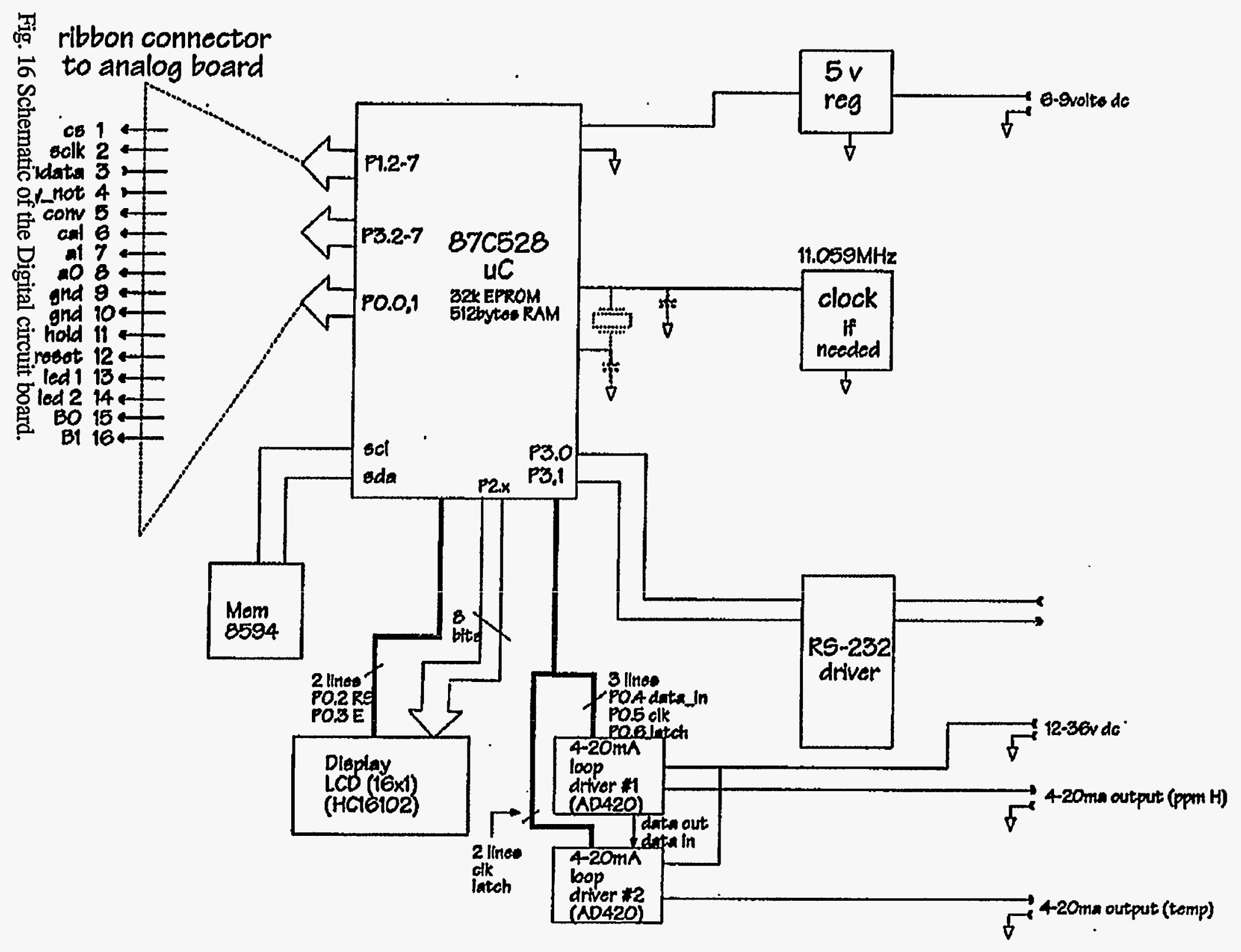




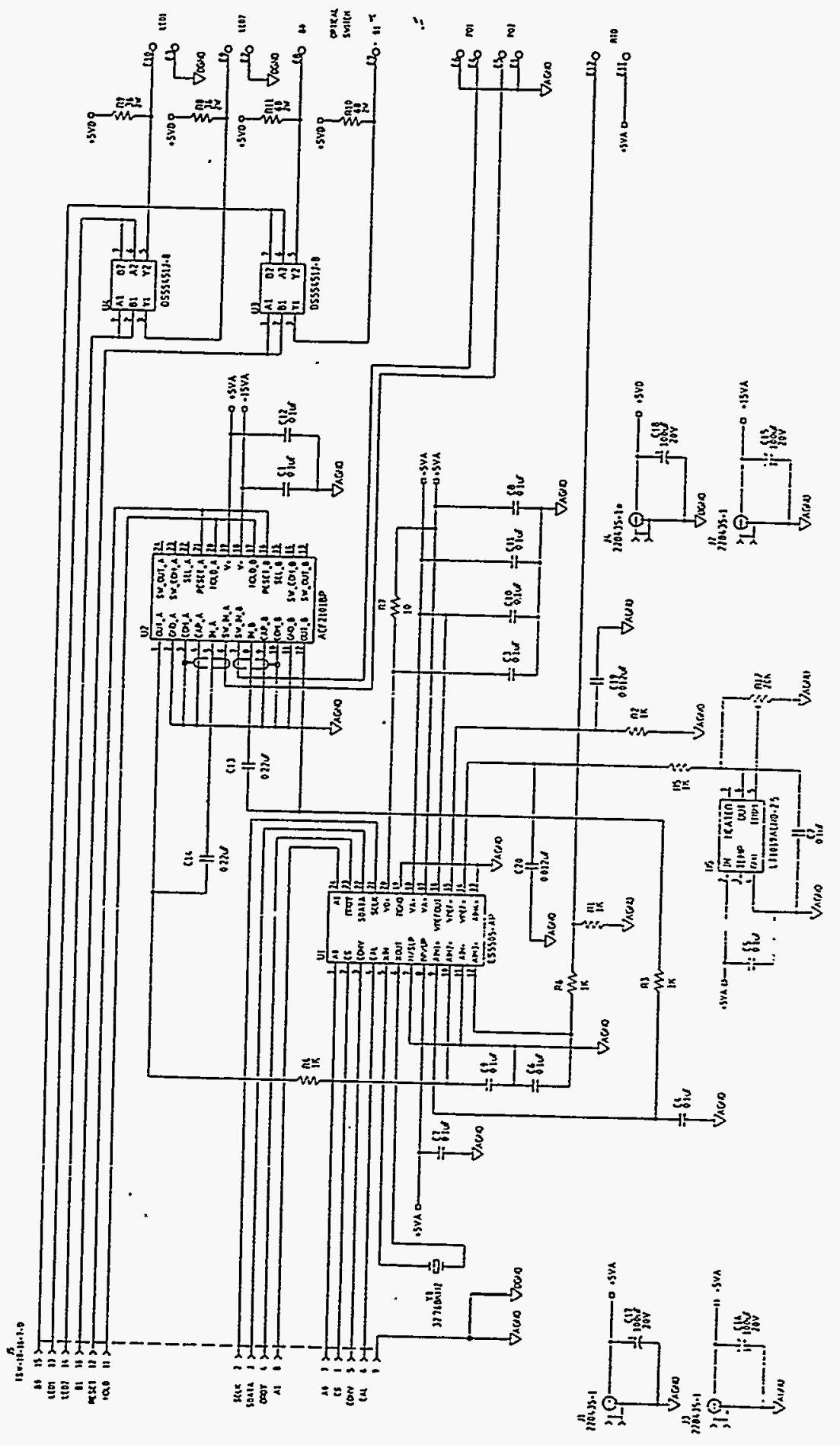

Fig. 17 Analog board schematic. 
LED

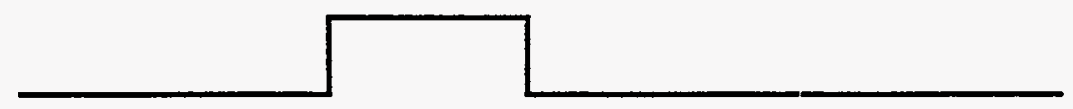

Hold A \& B

Reset A \& B

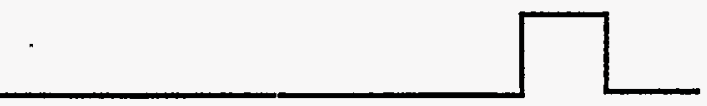

Convert A

\section{Convert B}

Fig. 18 Measurement timing sequence for prototype instrument.

The package can be run in auto or command mode. In auto mode the instrument continuously makes measurements of hydrogen concentration ands temperature and outputs these calibrated values. In command mode individual operations must be sent to the micro-processor via the RS-232 port. The commands are described in the discussion of the software. The data output from the RS-232 port is un-processed and needs to be manipulated. Command mode is mainly used for troubleshooting.

\section{Using the Harley System Software}

Upon power-up the software goes through a initialization code which initializes variables, the display, 4-20 ma D/A's, takes some dummy A/D readings, and reads permanent memory (if present). After the initialization routines the system enters the "main" loop. This loop performs the data taking operation, computation and output formatting / presentation. This loop will run endlessly unless interrupted by a RS-232 input signal. The input signal is generated by sending any transmission to the RS-232 port.

Once a RS-232 transmission is sensed the program enters the "command" loop and waits for further RS-232 transmissions which will be interpreted as commands. Two kinds of input will result in action from the command function; 2 letter commands or input starting with a ".". The 2 letter commands will be executed immediately after interpretation and will result in the desired action. After completion of the command, the system is left in the command mode (unless the command is "ex" which will restart continuous system operation). If the input string starts with ":" then the command mode will analyze (parse) the string after a terminating "return", and perform the 
required action. The ":" commands are used to set variable values (which will be stored in permanent memory) or to set up a "execution sequence" for the "es" command.

The microcontroller code also includes the use of a watchdog timer. The use of a watchdog enables the microcontroller module to reset itself if any operation does not operate properly (in which case the watchdog would time out and reset the microcontroller). Each section of code contains the code element $\{\mathrm{T} 3=0 ;$ WDCON $=0 \times 5 \mathrm{a} ;\}$, this element resets the watchdog timer and starts the watchdog. Any section of code which fails to do the reset operation (meaning the code has encountered a catastrophic problem) will cause the system to reset and restart.

\section{The "2 letter commands"}

Shown below is a copy of the main menu of commands present in the Harley system. These commands are generally used to troubleshoot problems within the system and allow the operator to access raw data.

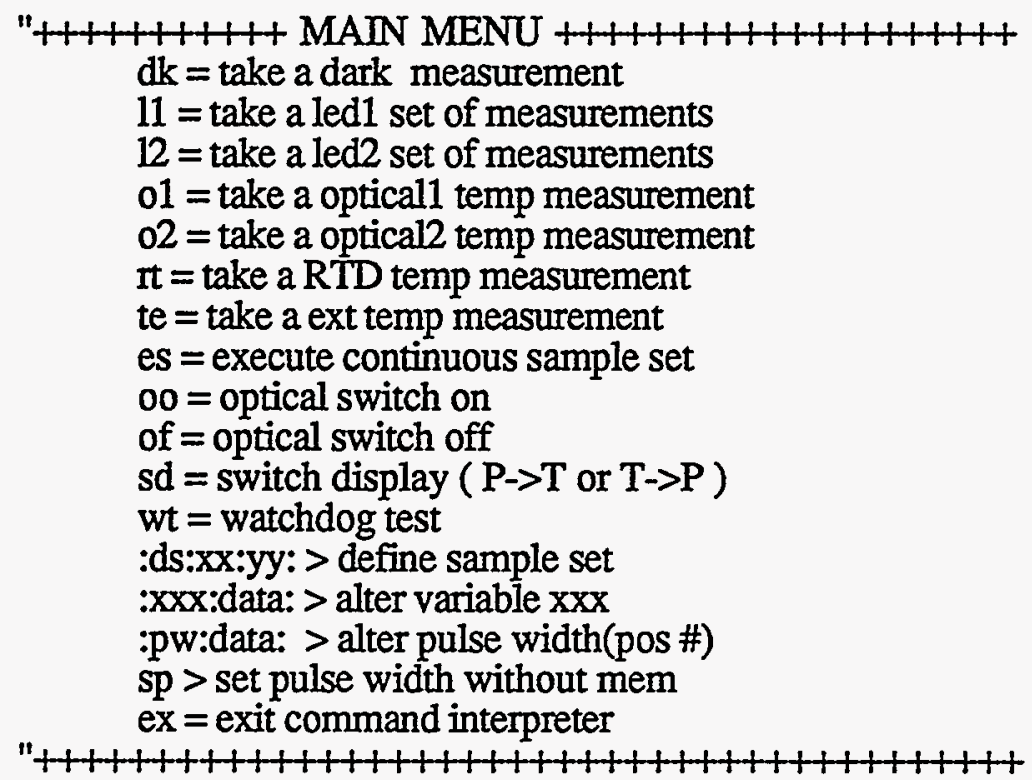

The first 7 commands (dk,11,12,01,02,rt and te ) cause the system to take the associated readings and "display" them on the RS-232 port. Two of the other commands $(00$, of $)$ control the state of the optical switch. The "es" command causes the system to cycle through a defined sample set composed of several.of the first seven commands (the sample set is defined by use of the :ds:xx:yy:... command - described below). The sd command toggles the LCD display output' which is used when the system is in continuous run mode (normal operating mode). If the display was in pressure then it changes to temperature, if it was in temperature then it will "toggle" to pressure. On reset the LCD display will come up displaying pressure. The "wt" command tests the watchdog timer operation by putting the system into a infinite loop and waiting for the watchdog to time out and restart the system. After executing a "wt" the system reverts to normal run mode. If the system does not restart then something is wrong with the watchdog. Using the sp command (followed by inputting the data) will change the optical output pulse width but will not memorize the value. This value will stay in force until the system is reset or until it is changed again. This command should not be used in general (one should use the ":" command to change this variable, i.e. ":pw:data:"). The "ex" command will cause the system to exit the command menu and enter the continuous run mode. Another command which is not listed on the menu above is the "st" command. "st" will display the alterable variable list and values. This can be useful when changing variable values using the ":variable:data:" construct shown below. 
The ":" (colon) command.

The ":" command set is used to change the operating and permanent value of system function and control variables. The command is used as follows; ":variable name:data:". The variable name can be any of the following; $\mathrm{m0t}, \mathrm{m} 1 \mathrm{t}, \mathrm{m} 2 \mathrm{t}, \mathrm{m} 3 \mathrm{t}, \mathrm{m} 4 \mathrm{t}, \mathrm{m} 0 \mathrm{p}, \mathrm{m} 1 \mathrm{p}, \mathrm{m} 2 \mathrm{p}, \mathrm{m} 3 \mathrm{p}, \mathrm{m} 4 \mathrm{p}$, $\mathrm{m} 5 \mathrm{p}, \mathrm{m} 6 \mathrm{p}, \mathrm{m} 7 \mathrm{p}, \mathrm{m} 8 \mathrm{p}, \mathrm{m} 9 \mathrm{p}, \mathrm{m} 10 \mathrm{p}, \mathrm{m} 11 \mathrm{p}$, or $\mathrm{pw}$.

The data field can be in integer or floating point (1e-3) format. " $\mathrm{m} 0 \mathrm{t}$ " $\rightarrow$ " $\mathrm{m} 4 \mathrm{t}$ " are external temperature function variables ( $T$ ext function in the program code). "m0p" $\rightarrow$ " $m 11 p$ " are hydrogen pressure function variables ( $\mathrm{H}$ _pres function in the program code). "pw" controls the optical pulse width in msec. It must be an integer from 1 to 65535 (msecs).

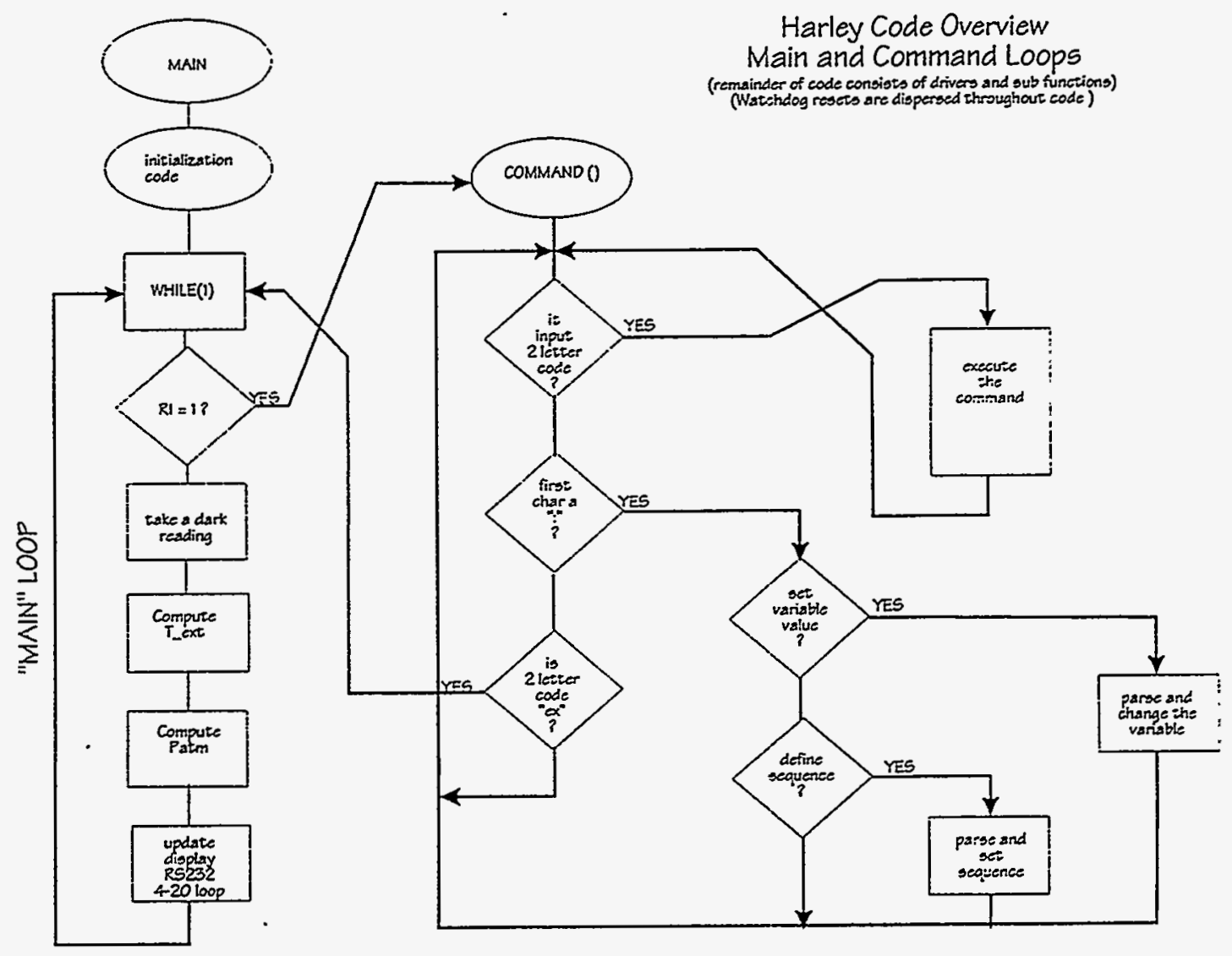

Fig. 19 Flow diagram for the microprocessor sottware.

The "." command is also used to set up a task sequence which will later be "executed" by the "es" command. A typical example of the use of the ":ds:" sequence is ":ds:rt:11:12:". This command will cause the system to continuously cycle, taking a internal temperature reading then a led 1 reading then a led 2 reading, when the "es" command is executed. The cycling will stop when any RS-232 transmission is received. This command is useful for looking at the raw data and for troubleshooting.

A copy of the software is presented in Appendix A. A flow diagram of the decision making process of the program is shown in fig. 19. While specialized for the particular problem of detecting hydrogen in transformer oil the instrument and software could relatively easily be adapted to chemical detection problems by use of other sensing coatings and appropriate changes in the measurement sequence and calibration functions. 


\section{Calibration Functions}

The instrument reads out in practical units for both hydrogen concentration and temperature. To do this it is necessary for the microprocessor to apply calibration functions to raw data. These functions, in addition to providing the calibration, also correct for the effects of variation in the intemal temperature and for the hydrogen sensor the extemal temperature. For the temperature sensor the following calibration function is used:

$$
T=22.1-\frac{1}{m_{3 t}}\left[m_{0 t}+m_{1 t} x+m_{2 t^{x}}{ }^{2}-R\right]+m_{4 t}
$$

where the temperature is in ${ }^{\circ} \mathrm{C}, \mathrm{R}=(\operatorname{Sig} 810 \operatorname{Ref} 880 / \operatorname{Sig} 880 \mathrm{Ref} 810)$, and $\mathrm{x}$ is the internal temperature in ${ }^{\circ} \mathrm{C}$. The $\mathrm{m}$ parameters are numbers that can be down loaded into the microprocessor through the RS-232 port using the software commands described above. The dependence on internal temperature comes from the variation in the electronics with temperature. Primarily, this is the variation in the wavelength of the LED sources with temperature which will affect the reflectivity of the temperature sensor. This dependence is fit with a simple quadratic function with the parameters $\left(\mathrm{m}_{0} \mathrm{t}, \mathrm{m}_{1} \mathrm{t}\right.$, and $\mathrm{m}_{2} \mathrm{t}$ ) determined by varying the temperature of the electronic package while keeping the sensor at $22.1^{\circ} \mathrm{C}$. The parameters ( $\mathrm{m} 3 \mathrm{t}$ and $\mathrm{m} 4 \mathrm{t}$ ) are the calibration parameters and are determined by varying the temperature of the sensor.

For the hydrogen sensor the behavior is more complex because the reflectivity of the palladium alloy film depends on the sensor (external) temperature as well as the hydrogen concentration in a non-linear way. Thus the calibration function is much more complex and requires more parameters. The function is not derived theoretically but results from an empirical fit of the data. Thus, it probably is not unique. The following function is used:

$$
P_{a t m}=\left[\left\{\frac{m_{8 p}+m_{9 p} m_{11 p} Q+m_{10 p^{2} m_{11 p} Q^{2}}}{m_{7 p}-m_{8 p}+m_{9 p} m_{11 p} Q+m_{10 p} m_{11 p}^{2} Q^{2}}\right\} \frac{\exp \left(-\frac{m_{5 p}}{273.1+T}\right)}{m_{6 p}}\right]^{2}
$$

where

$$
\mathrm{Q}=\frac{\mathrm{m}_{0 p}+\mathrm{m}_{1 \mathrm{p}} \mathrm{x}+\mathrm{m}_{2 p \mathrm{x}^{2}+\mathrm{m}_{3 p}(22.1-\mathrm{T})+\mathrm{m}_{4 p}-\mathrm{R}}}{\mathrm{m}_{0 \mathrm{p}}+\mathrm{m}_{1 \mathrm{p}} \mathrm{x}+\mathrm{m}_{2 \mathrm{p}} \mathrm{x}^{2}+\mathrm{m}_{3 p}(22.1-\mathrm{T})+\mathrm{m}_{4 p}}
$$

and the hydrogen pressure is in atmospheres. Calibration is achieved in a similar manner to the temperature sensor with variation of the temperature of the electronics package while the sensor 
temperature and hydrogen concentration are held fixed followed by variation in both hydrogen concentration and temperature at a fixed electronics package temperature. Least squares fits are used to determine the optimum parameter values. With these calibration functions the instrument reads out in practical units of $\mathrm{ppm}$ for hydrogen concentration and ${ }^{\circ} \mathrm{C}$ for temperature. As with all sensors each one must be individually calibrated until a manufacturing method can be developed that guarantees the reproducibility of the sensor response. This is a very difficult problem to solve.

\section{Measurements in Transformer Oil}

The end use for the temperature and hydrogen sensors and the electronics package was to detect hydrogen in oil in large electrical transformers. Thus it was necessary to build a test system for evaluating sensor performance for detecting hydrogen dissolved in transformer oil. A schematic of the laboratory system is shown in Fig. 20. Used transformer oil is pumped through tygon tubing using a peristaltic pump (Cole-Parmer Masterflex) with a range of flow rates. The oil is extracted from a reservoir and pumped through a tee shaped glass tube containing the sensor. The oil then returns to the reservoir. Various gases are dissolved in the oil by bubbling them through the oil in the reservoir using a tube terminated in a glass frit. The oil temperature can also be changed since the oil reservoir is on a Thermolyne hotplate. The temperature of the oil is measured with a thermocouple. With this system the amount of dissolved hydrogen in the oil can be varied and even replaced by air or other suitable gas.

This system was used to evaluate the stability and sensitivity of palladium-silver alloy coating for sensing dissolved hydrogen gas. Measurements were made over the temperature range $20-120^{\circ} \mathrm{C}$ and gas concentrations as low as $50 \mathrm{ppm}$. No evidence of degradation of response was observed. Measurements of $100 \mathrm{ppm}$ concentration of hydrogen in oil were made over a one month period without removal of the sensor from the oil with no indicated change in response. These measurements were made with palladium-silver alloy coatings deposited directly on the end of an optical fiber as opposed to the microlens coatings finally used toward the end of this program. Thus these results are not predictive of the performance of the final multi-coating structure films. However, they do indicate that stable sensing films are feasible. Further development of the coatings to relieve stresses while maintaining speed, sensitivity, and stability in the operating environment is necessary. While this test system provided a reasonable test of sensor viability in hot transformer oil, it must ultimately be supplemented by real field tests so that the effects of any unexpected variables can be evaluated. Unfortunately, the pace of development of the instrument did not allow field testing in the 3 year program. It is thought that further coating development will be necessary before field testing can be successfully undertaken. A typical response of the instrument to $50 \mathrm{ppm}$ of hydrogen dissolved in transformer oil followed by air is shown in Fig. 21. 


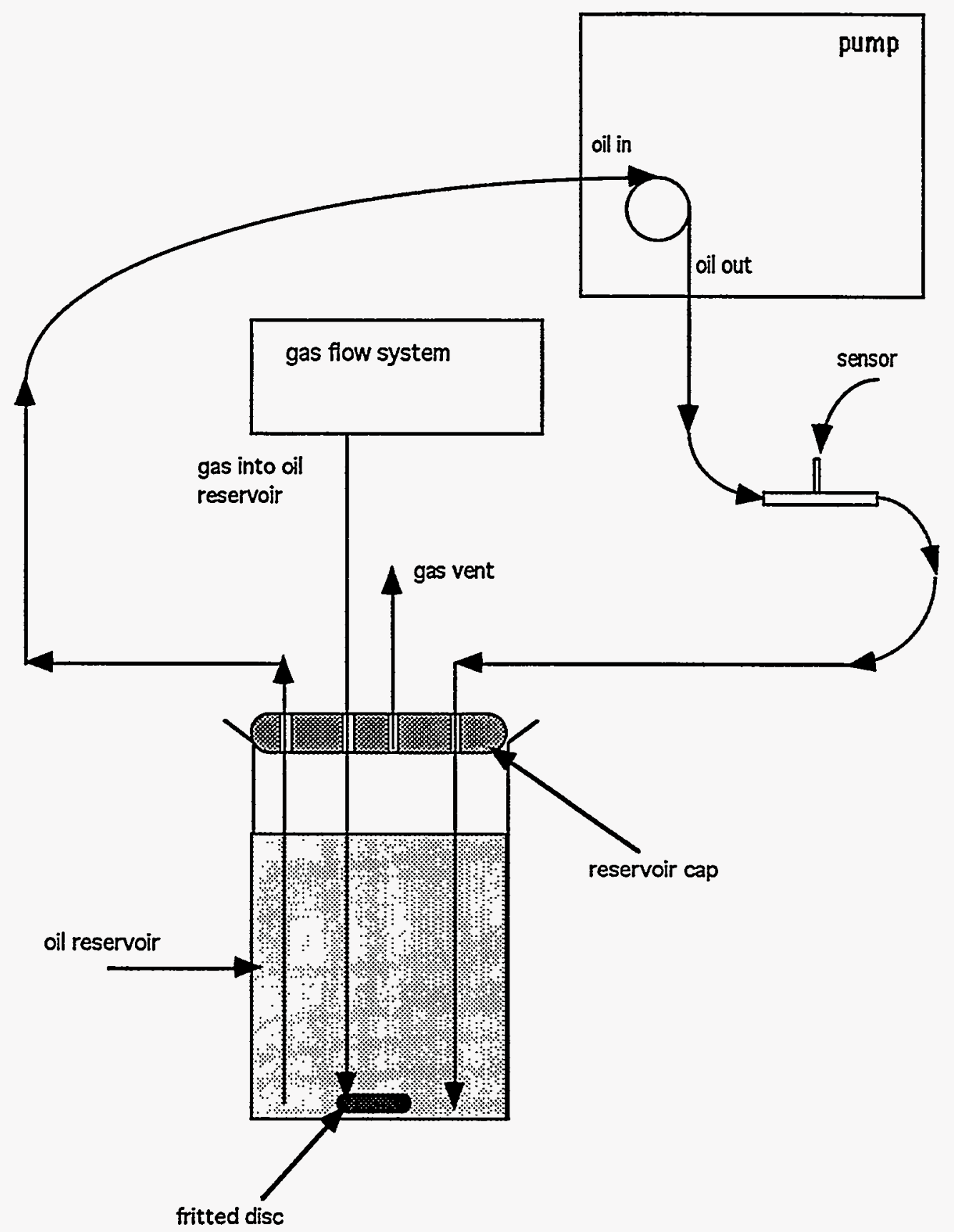

Fig. 20 Test system for sensor measurements in transformer oil. 


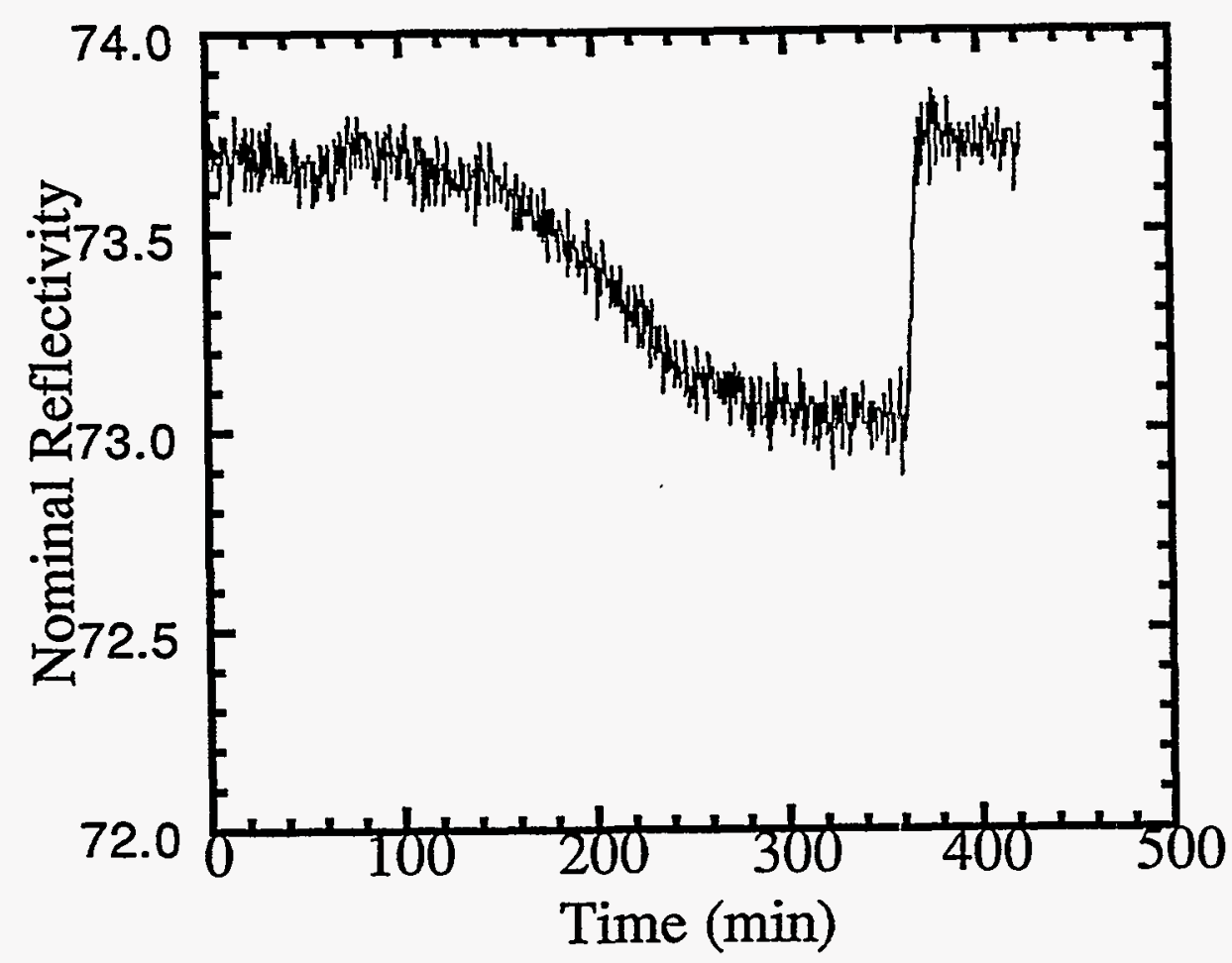

Fig. 21 Sensor response to $50 \mathrm{ppm} \mathrm{H}_{2}$ in transformer oil followed by air at room temperature.

\section{Commercialization Potential}

The ultimate goal of the project was to develop a prototype instrument that might be commercializable. This would depend not only on performance of the instrument but also cost, manufacturability, and ease of use. Cost and manufacturability depends to a large extent on the complexity of the instrument. We are fortunate that the current version of the design has a limited number of parts. This will limit costs as well as assist in the manufacturability of the instrument. Excluding R\&D and design costs, the electronics package and sensors, in quantity, could be quite cost effective. When purchasing components in small quantities, the total cost of the electronics package, optical board, enclosures, and all internal optical and electrical connections would come to less than $\$ 1500$. If the components were purchased in wholesale quantities, considerable savings would be possible. Furthermore, the continued development of the optical components will reduce costs even further. Packaging will depend in detail on the application and design specifics. The breakdown of parts and cost is as follows.

\section{Optical Board}

IC Temperature Transducer

LED $(810 \mathrm{~nm})$

LED $(900 \mathrm{~nm})$

Photo Diode
(1) $\$ 2.21$

(1) $\$ 146.00$

(1) $\$ 67.00$

(2) $\$ 32.00$ ea
$\$ 2.21$

$\$ 146.00$

$\$ 67.00$

$\$ 64.00$ 
Optical Switch

$1 \times 2$ Coupler

$2 \times 2$ Coupler

SMA Optical Connector

FC/PC Optical Connector

TOTAL
(1) $\$ 250.00$

(1) $\$ 47.00$

(1) $\$ 47.00$

(4) $\$ 10.06$ ea

(2) $\$ 17.00 \mathrm{ea}$
$\$ 250.00$

$\$ 47.00$

$\$ 47.00$

$\$ 40.24$

$\$ 34.00$

$\$ 697.45$

\section{Analog Board}

$32.768 \mathrm{KHz}$ Crystal

Voltage Reference

Dual Driver

Dual Switched Integrator

A/D Converter

20KOHM Potentiometer

68 OHM Resistor

36 OHM Resistor

10 OHM Resistor

$1 \mathrm{~K}$ OHM Resistor

16 pin Terminal Strip

SMB Connector

$0.012 \mathrm{uF}$ Capacitor

100uF Capacitor

0.22uF Capacitor

$0.10 u F$ Capacitor

Custom PC Board

24 pin Dip Socket

8 pin Dip Socket

TƠTAL

\section{Digital Board}

87C528 microprocessor

PC board

8594 memory

4-20 ma loop drivers

RS-232 driver

11.059 MHz clock

$5 \mathrm{v}$ regulator

LCD display

Miscellaneous parts

TOTAL
(1) $\$ 0.70$

(1) $\$ 7.00$

(2) $\$ 2.00 \mathrm{ea}$

(1) $\$ 22.00$

(1) $\$ 23.00$

(1) $\$ 0.50$

(2) $\$ 3.00$ ea

(2) $\$ 3.00 \mathrm{ea}$

(1) $\$ 0.05$

(6) $\$ 0.16 \mathrm{ea}$

(1) $\$ 0.50$

(4) $\$ 5.88$ ea

(2) $\$ 0.25$ ea

(4) $\$ 4.75$ ea

(2) $\$ 0.40$ ea

(12) $\$ 0.23$ ea

(1) $\$ 120.00$

(2) $\$ 3.50 \mathrm{ea}$

(3) $\$ 0.47 \mathrm{ea}$
$\$ 0.70$

$\$ 7.00$

$\$ 4.00$

$\$ 22.00$

$\$ 23.00$

$\$ 0.50$

$\$ 6.00$

$\$ 6.00$

$\$ 0.05$

$\$ 0.96$

$\$ 0.50$

$\$ 23.52$

$\$ 0.50$

$\$ 19.00$

$\$ 0.80$

$\$ 2.76$

$\$ 120.00$

$\$ 7.00$

$\$ 1.41$

$\$ 245.70$

$\$ 65.00$

$\$ 40.00$

$\$ 4.00$

$\$ 40.00$

$\$ 8.00$

$\$ 10.00$

$\$ 0.30$

$\$ 24.00$

$\$ 10.00$

$\$ 201.30$

Power supply modules and aluminum chassis box add about $\$ 200.00$.

The overall cost is thus compatible with the envisioned market of a sensor package for monitoring hydrogen in transformer oil. By changing sensor coatings this prototype instrument can also be used to monitor other chemical species. An optical switch with more ports will also allow monitoring of a large array of sensors using the same basic instrument design. This flexibility will make the instrument useful for other applications. Thus it appears that cost will not be a limiting factor in the commercialization of this type of fiber optic chemical sensor. 


\section{Summary}

At the beginning of this project a fiber optic hydrogen sensor existed in the laboratory that had limited sensitivity, operated only in gas at room temperature, and was a laboratory experiment (i.e. not an instrument). The goals of the project where to extend the development of this sensor to achieve the high sensitivity and operation in oil at elevated temperatures necessary for monitoring dissolved hydrogen in transformer oil. Furthermore, if this sensor proved feasible to develop a prototype instrument would be developed that could be used for field testing. Palladium-silver alloys with enhanced solubility of hydrogen were shown to greatly enhance the sensitivity of the sensor and this coating material was also used successfully in used transformer oil over the temperature range $20^{\circ} \mathrm{C}-120^{\circ} \mathrm{C}$. Exposure of the sensor to other gases showed that only oxygen at concentrations at least 10 times higher that the hydrogen concentration interfered with the response of the sensor. With these encouraging results a prototype instrument was designed to monitor hydrogen in transformer oil. A temperature sensor that utilizes the same instrument package was also developed as proper interpretation of the hydrogen sensor response requires a knowledge of the sensor's temperature. The instrument package is a generic design in that sensing of other chemical species is possible with the same package by utilizing appropriately coated fiber ends as sensors. By using a larger optical switch arrays of sensors could also be monitored.

While a field test of the prototype instrument was not achieved due to reliability problems of the sensors caused by process control of the coating depositions, the underlying concept for monitoring hydrogen in transformer oil was proved viable. Furthermore, the instrument developed can contribute to other potential applications of this technology by providing a practical plafform for an array of potential chemical sensors.

\section{Acknowledgments}

This work was performed at Sandia National Laboratories supported by the U.S. Department of Energy under contract DE-AC04-94AL8 5000. The authors would like to acknowledge Allyn Anderson, 2235 for layout and assembly of the digital circuit board. The authors are indebted to the employees of J. W. Harley, Inc. for their collaboration on this project and their insight and knowledge of the operating conditions and requirements for a hydrogen sensor for transformer oil monitoring. We are particularly indebted to Richard Berris for his input to the design of the prototype instrument. 


\section{REFERENCES}

1. M. A. Butler, J. Electrochem. Soc. 138, L46 (1991).

2. M. A. Butler, Sensors and Actuators B 22, 155 (1994).

3. M. A. Butler \& A. J. Ricco, Appl. Phys. Letters 53 (1988) 1471.

4. M. A. Butler, A. J. Ricco \& R. J. Baughman, J. Appl. Phys. 67 (1990) 4320.

5. Hydrogen in Metals II edited by G. Alefeld and J. Volkl (Springer, New York, 1978).

6. K. Allard, A. Maeland, J. W. Simons \& T. B. Flanagan, J. Phys. Chem. 72, 136 (1968).

7. H. Brodowsky \& E. Poeschel, Z. Phys. Chem.NF 44, S143 (1965).

8. Optical Fiber Sensors Vol. II edited by B. Culshaw \& J. Dakin (Artech House, Norwood, MA, 1989) p. 662.

9. L. Shultheis, H. Amstutz \& M. Kaufmann, Optics Letters 13, 782 (1988).

10. G. Beheim, K. Fritsch, \& M. T. Azar, SENSORS January (1990) pg. 37.

11. J. W. Berthold, S. E. Reed, \& R. G. Sarkis, Optical Engineering 30, 524 (1991). 


\section{APPENDIX A}

\% Code developed by George Dulleck, Dept 2254 Sandia National Laboratories*/

\#include <c:lc51linclreg528.h>

\#include <c:lc51linclstdio $h>$

\#include <c: $\mid c 51$ linclstdlib.h $>$

\#include <c: $\mid c 51$ inclintrins.h>

\#include <c:lc51linclstring.h $>$

\#include <c:lc51linclabsacc.h>

\#include <c:lc51linclmath.h>

\#include <c:lc51 lexamples 528 real.h $>/ *$ alter build memory space */

\#pragma MAXARGS(20)

\#define uint unsigned int

\#define uchar unsigned char

\#define rtemp 4

\#define dark $3 / *$ no led's on */

\#define on $1 / *$ "on" $=1 *$ /

\#define off $0 / *$ "off" $=0 *$ I

\#define high 1

\#define low 0

\#define ref 1

\#define sig 0

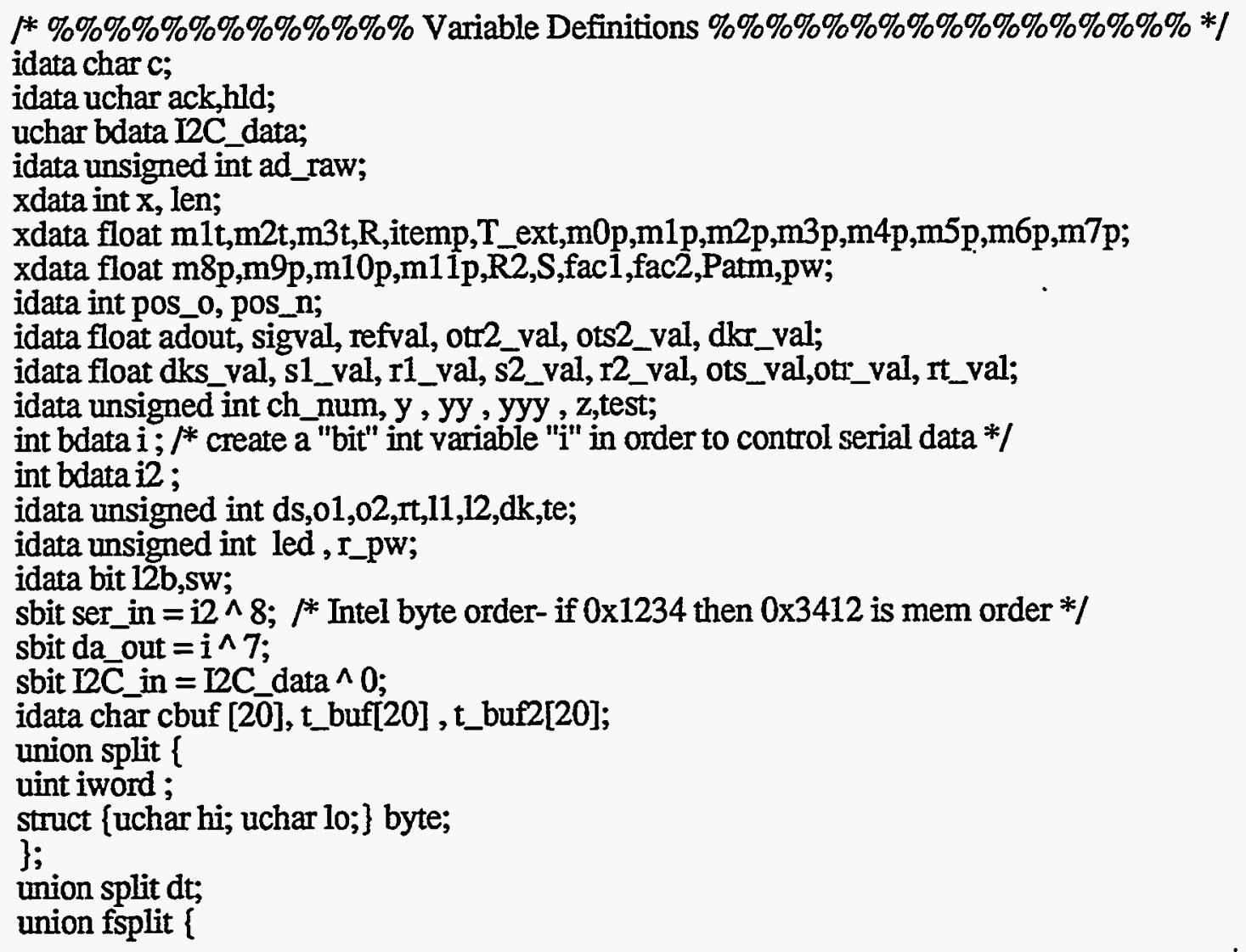


float fword;

struct [uchar hi; uchar hmid; uchar Imid; uchar lo; \} byte;

\};

union fsplit xdata $\mathrm{m0t}$, m4t,stored;

/* \%\%\%\%\%\%\%\%\%\%\%\% End of Variable Definition \%\%\%\%\%\%\%\%\%\%\%\%\%\%\%*I

$j^{*} \% \% \% \% \% \% \% \% \% \% \% \%$ Redefine I/O kemals for use with WatchDog \%\%\%\%\%\%*I char_getkey 0 \{

char c;

while (!RD) $\{\mathrm{T} 3=0 ; \mathrm{WDCON}=0 \times 5 \mathrm{~A} ;\} \quad / *$ adding $\mathrm{T} 3$ reset is the only change $*$ /

$\mathrm{c}=$ SOBUF;

$\mathrm{RI}=0$;

\}

return (c);

\#define XON Ox11

\#define XOFF 0x13

char putchar (char c) \{

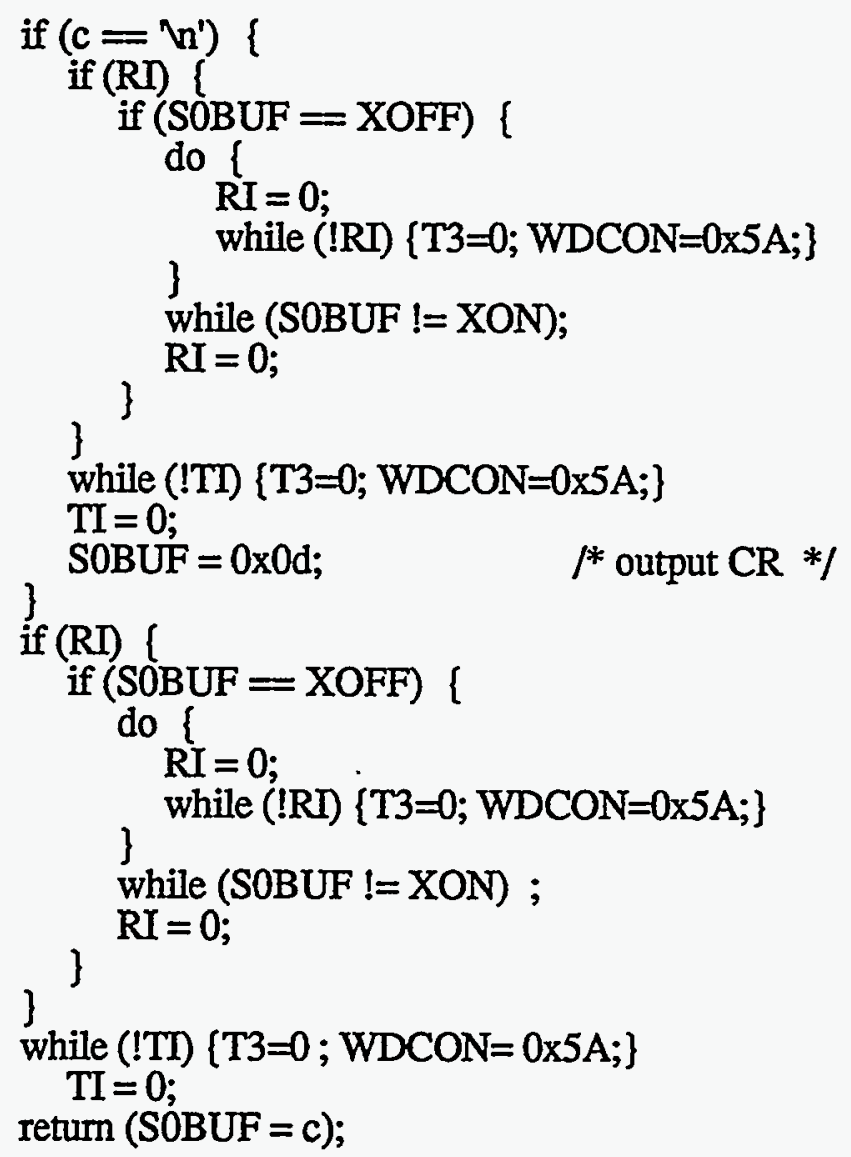

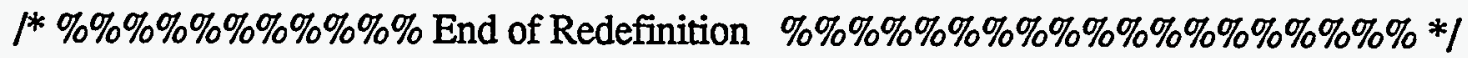
/* \%\%\%\%\%\%\%\%\%\%\% Start of Code Area \%\%\%\%\%\%\%\%\%\%\%\%\%\%\%\%\%\%\%*I code char cwst[] = "n" 
" Copyright 1995/n"

"Sandia National Laboratories/George Dulleckin";

code char mmenu[] =

" $\mathrm{n}$ "

" $+++1++1+++$ MAIN MENU $+1++1++++++++++1++1-\mathrm{n}^{\prime \prime}$

"I $\mathrm{dk}=$ take a dark measurement $+\mathrm{n}$ "

"l $11=$ take a led1 set of measurements $+n^{\prime \prime}$

"I $12=$ take a led 2 set of measurements $+n^{\prime \prime}$

"l 01 = take a opticall temp measurement $+\backslash n$ "

"I 02 = take a optical2 temp measurement $+\backslash n$ "

"I $\mathrm{rt}=$ take a RTD temp measurement $+\mathrm{n}$ "

"l te $=$ take a ext temp measurement $+n^{\prime \prime}$

"l es = execute continuous sample set $+\mathrm{hn}^{\prime \prime}$

"I $00=$ optical switch on + "n"

"l of = optical switch off $\quad+\mathrm{n}^{\prime \prime}$

"I st = status, list variable values $+\mathrm{ln}^{\prime \prime}$

"I $s d=$ switch display $(P->T$ or $T->P)+(n "$

"I $\mathrm{wt}=$ watchdog test

"l :ds:xx:yy: > define sample set $+\mathrm{hn}^{\prime \prime}$

"I :xxx:data: > alter variable $\mathrm{xxx}+\mathrm{n}^{\prime \prime}$

"l :pw:data: > alter pulse width(pos \#) +

"l sp > set pulse width without mem + $\mathrm{ln}$ "

"l $\mathrm{ex}=$ exit command interpreter $+\mathrm{n}$ "

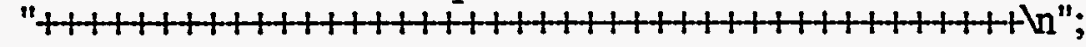

void dt_exe $(\mathrm{pw})\{/ *$ time delay function using software loop */

$l^{*}$ timer 0 was used prior to software loop, commented out now */

* extem idata unsigned int $\mathrm{pw} ; *$ I

* printf ("address of pw = \%bx in", (char) \&pw ); *I

// dt.iword $=0 \mathrm{xFFFF}-\mathrm{pw} * 922 ; / *$ calculate timer load value $* /$

// THO = dt.byte.hi;

// TLO = dt.byte.10;

// $\mathrm{TF} 0=0$

$/ /$ TRO $=1 ; / *$ start timer $0 * /$

// while (TF0 $=0$ ); $/ *$ wait for timer to overflow */

$/ / \mathrm{TRO}=0 ; / *$ stop timer $0 *$ /

yyy $=0$;

test $=($ uint $)\left(\mathrm{pw}^{*} 40\right)$;

while (yyy < test) $\{\mathrm{T} 3=0 ; \mathrm{WDCON}=0 \times 5 \mathrm{~A} ;$ yyy++; $\}$

\}

void cal_exe(void)

cal $=1 ; /^{*}$ produces time delay in the cal output */

$\mathrm{cal}=1$;

$\mathrm{cal}=1$;

$\mathrm{cal}=1$;

$\mathrm{cal}=0$

cal $=0$

cal $=0$;

\}

void ad_exe (ch_num $)\{\quad \rho$ A/D control and read *I 
$\{\mathrm{T} 3=0 ; \mathrm{WDCON}=0 \times 5 \mathrm{~A} ;\}$

$\mathrm{y}=1$;

while $(y<3)\{$

i2 $=0$;

ad_raw $=0$;

cs $=1$;

al $=0$;

if (ch_num $==$ sig) $\mathrm{a} 0=0$;

if (ch_num $=$ ref) $\mathrm{a} 0=1$;

$\mathrm{a} 0=0$

if (ch_num $=$ itemp) [

a1 =

\}

dt_exe(20);

[T3=0; WDCON $=0 \times 5 \mathrm{~A} ;\}$

conv $=1$;

cal_exe0;

while (drdy_not $=1) ; / * \mathrm{P} 4=0 \times 00 * /$

cs $=0$;

conv $=0$;

\{T3=0; WDCON $=0 \times 5 A ;\}$

for $(x=1 ; x<=16 ; x++)(\quad / *$ read the A/D data-serially $*$ I

i2 $=0$;

ser_in = sdata;

ad_raw $=$ ad_raw $+\mathrm{i} 2$;

sclk $=1$

ad_raw = irol_(ad_raw, 1);

$\mathrm{sclk}=0 ; F^{*}$ use the inst above as a time delay $*$ I

\}

adout $=$ ad_raw;

[T3=0; WD CON $=0 \times 5 \mathrm{~A}$;

if (ad_raw $<=0 \times 2800$ ) adout $=$ adout $/ 65536 * 2.5$;

if $($ ad_raw $>0 \times 8000)$ \{ adout $=\sim($ ad_raw $)$;

adout $=$ adout $/ 65536 *-2.5$;

\}

if (ad_raw $>0 \times 2800 \& \&$ ad_raw $<=0 \times 8000)$ adout $=-1.250-(0 \times 8000-$ ad_raw $) * 2.5 / 65536$; $y++$

$/ / \mathrm{uC}$ code after 8/8/95 will use the code below to replace above

// for $(x=1 ; x<=16 ; x++)\{\quad *$ read the A/D data-serially */

//ad_raw_=_irol_(ad_raw,1);

$/ / 12=0$;

//ser_in = sdata;

//ad_raw = ad_raw $+\mathrm{i} 2$;

//scik $=1$;

//delay();

//delayO;

//sclk $=0 ; / *$ use the inst above as a time delay */

II]

// adout2 = ad_raw;

$/ /$ adout $2=($ adout $2-0 \times 8000) / 65536 * 5$;

\} 
if (ch_num = sig) sigval = adout;

if (ch_num = ref) refval = adout;

if (ch_num = Itemp) rt_val = adout;

[T3=0; WDCON=0x5A; $\}$

\}

void resett $(\mathrm{pw})\{\quad \stackrel{*}{*}$ reset pulse generator */

reset $=$ off;

dt_exe(pw);

dt_exe(pw);

reset $=$ on;

]

void led_exe (pw,led) [ $/ *$ LED pulse generator */

if $($ led $=1$ ) led $1=$ on;

if (led $=$ dark);

if (led $=2$ ) led2 = on;

dt_exe(pw);

le $\bar{d} 1=$ led2 $=$ off;

\}

void process ( led ) $\{\quad / *$ "process" does one sig/ref set using led $\mathrm{x} *$ I

resett(50);

hold = off;

if (led = dark) led_exe(pw,dark);

if $($ led $=1)$ led_exe $(\mathrm{pw}, 1)$;

if $($ led $=2$ ) led_exe(pw,2);

hold $=$ on ;

ad_exe (sig);

ad_exe (ref);

resett(50); $/ *$ leaves integrators in a discharged state */

$f^{*}$ sig is on analog 1 ie $00 *$ /

$\rho^{*}$ ref is on analog 2 ie $01 *$ I

* temp is on analog 3 ie $10 *$ /

* analog 4 is not used */

\}

void st_exe(pw) $\{\quad / *$ compute dt word and bytes and display */

dt.iword $=\mathrm{pw} * 922$;

\}

void $\mathrm{PE}$ (void) $\{/ *$ Pulse $\mathrm{E}$ - generates the pulses for lcd line $\mathrm{E} *$ /

$E=$ on;

dt_exe(4);

$\mathrm{E}=$ off;

dt_exe(4);

) 
void $\mathrm{RH}$ (void)\{ $\not *$ Return the lcd cursor to home position */

$\mathrm{RS}=0$;

$\mathrm{DT}=0 \times 02$;

$\mathrm{PE}()$;

\}

void $F_{\text {_set(void) }}$ \}

$\mathrm{RS}=\overline{0}$;

DT $=0 \times 38 ; \quad / * 38$ for 2 line display; 30 for 1 line display */

$\mathrm{PEO}$;

\}

void D_off(void) \{

$\mathrm{RS}=\overrightarrow{0}$

$\mathrm{DT}=0 \mathrm{x} 0 \mathrm{C}$;

PEO;

\}

void mode_set(void)\{

$\mathrm{RS}=0$;

$\mathrm{DT}=0 \mathrm{x} 02$;

$\mathrm{PE}($;

\}

void sec_line(void) \{

$\mathrm{RS}=0$;

$\mathrm{DT}=0 \mathrm{xC} 0$;

$\mathrm{PE}($ );

] void $\mathrm{CD}$ (void) \{

$\mathrm{RS}=0$;

$\mathrm{DT}=0 \mathrm{x} 01$;

$\mathrm{PE}()$;

\}

void dis_lcd(void)[

RH(); $/^{*}$ return LCD cursor to home *I

$\mathrm{CD}() ; /$ * clear display *I

yy $=0$;

|* printf (":lcd:\%s $\backslash n "$, t_buf); */

RS = 1;

$\mathrm{12} \mathrm{b}=\mathrm{on}$;

[T3=0; WDCON $=0 \times 5 \mathrm{~A} ;\}$

while (yy<= strlen(t_buf)-1) $\quad / *$ transmit string to the LCD *I

if $(y y>=8 \& \& 12 b=$ on $)$ [sec_line $0 ; R S=1 ; 12 b=$ off; $\}$

DT $=$ t_buf $[y y]$

$\mathrm{PE}() ;$

yy+t;

$\{\mathrm{T} 3=0 ; \mathrm{WDCON}=0 \times 5 \mathrm{~A} ;\}$

$\mathrm{RS}=0$;

RH();

\} 


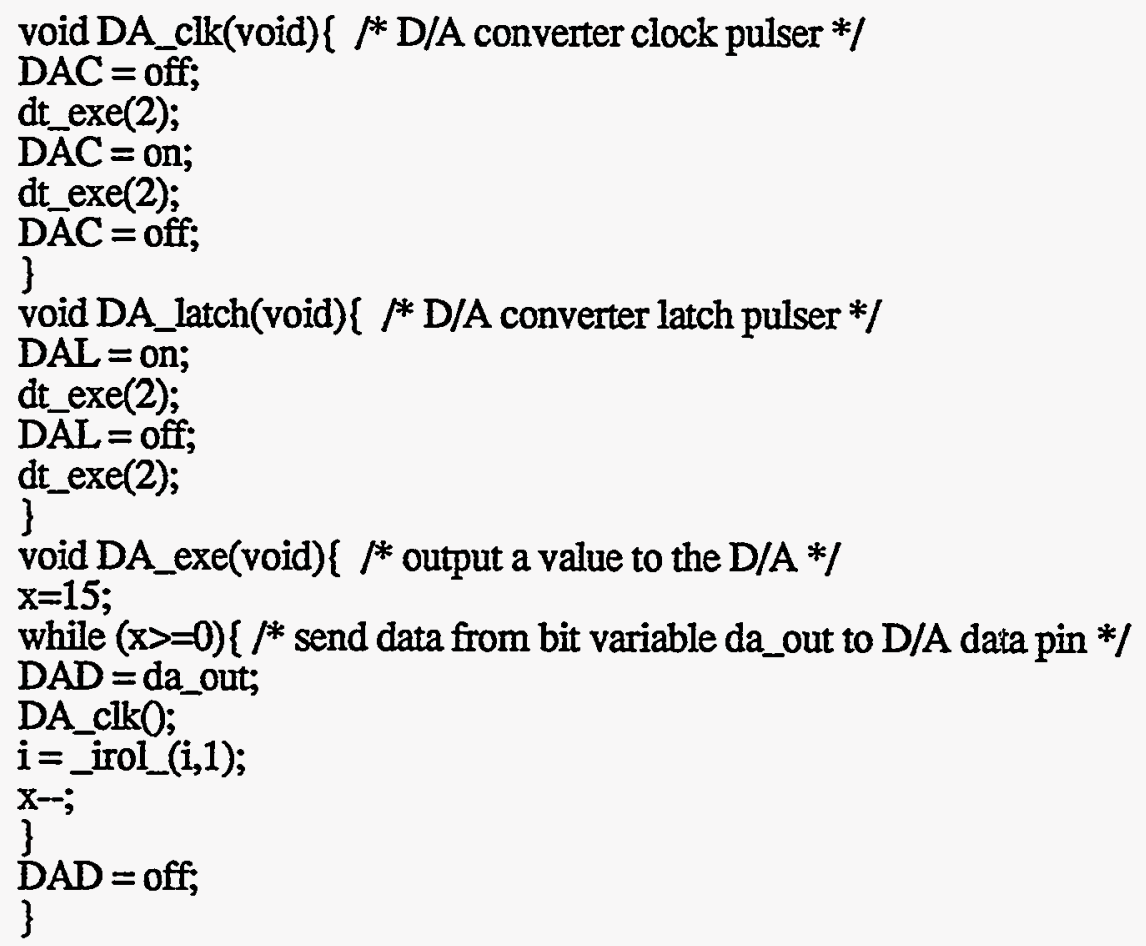

void temp_exe(void)\{ $/ *$ optical temperature function */ ad_exe(rtemp); 
void Hpres_exe(void) \{ $/ *$ pressure calculation function *]

o_switch(on);

process(1);

s1_val=sigval-dks_val;

r1_val=refval-dkr_val;

process(2);

s2_val=sigval-dks_val;

I2_val=refval-dkr_val;

o_switch(off);

\{ $33=0$; WDCON $=0 \times 5 A ;\}$

$\mathrm{R} 2=\mathrm{s} 1 \_\mathrm{val} * \mathrm{r} 2 \_\mathrm{val} /\left(\mathrm{r} 1 \_\mathrm{val} * \mathrm{~s} 2 \_\mathrm{val}\right)$;

$\{\mathrm{T} 3=0 ;$ WDCON $=0 \times 5 \bar{A} ;\}$

fac1 $=m 0 p+m 1 p *$ itemp+m2p*itemp*itemp+m3p*(22.1-T_ext)+m4p;

$\{\mathrm{T} 3=0 ; \mathrm{WDCON}=0 \times 5 \mathrm{~A} ;\}$

$S=(-R / f a c 1)+1$

[T3=0; WDCON $=0 \times 5 A$; ]

fac $2=m 9 p * m 11 p^{*} S+m 10 p * m 11 p * m 11 p * S * S ;$

$\{\mathrm{T} 3=0 ; \mathrm{WDCON}=0 \times 5 \mathrm{~A} ;\}$

$\mathrm{Patm}=((\mathrm{m} 8 \mathrm{p}+\mathrm{fac} 2) /(\mathrm{m} 7 \mathrm{p}-\mathrm{m} 8 \mathrm{p}-\mathrm{fac} 2)) *\left(\exp \left(-\mathrm{m} 5 \mathrm{p} /\left(273.16+\mathrm{T} \_\mathrm{ext}\right)\right)\right) / \mathrm{m} 6 \mathrm{p} ;$

$\{\mathrm{T} 3=0 ; \mathrm{WDCON}=0 \times 5 \mathrm{~A} ;\}$

Patm=Patm*Patm;

$\{$ T3=0; WDCON=0x5A;

void T_dis(void) \{

temp_exe);

printf ("R:\%+6.4e:T_ext:\%+7.5f",R,T_ext);

sprintf (t_buf, "T_ext $=\%+6.2 \mathrm{f} C$ ", T_ext);

dis_lcd0;

\}

void otemp_dis(void) $\{\quad *$ display a optical temp reading *

process(1);

ots_val = sigval - dks_val;

otr_val $=$ refval - dkr_val;

printf (":otemp1:\%+7.5f:\%+7.5f", ots_val, otr_val);

\}

void otemp2_dis(void) \{

process(2);

ots2_val = sigval - dks_val;

otr2_val = refval - dkr_val;

printf (":otemp2:\%+7.5f:\%+7.5f", ots2_val, otr2_val);

\}

void dk_dis(void) $\{\quad / *$ display dark value */

process(dark);

dks_val = sigval;

dkr_val = refval;

printf(":dark:\%+7.5f:\%+7.5f",dks_val,dkr_val);

\}

void $p 1$ dis(void) $[\quad / *$ display led1 values *I

process(1);

s1_val = sigval - dks_val; 
I1_val = refval - dkr_val;

printf (":led1:\%+7.5f:\%+7.5f",(float) s1_val,(float) r1_val);

sprintf( t_buf, "S1 = \%7.5f",s1_val); $/ *$ form str for lcd */

dis_lcdO;

\}

void p2_dis(void) $\{/ *$ display led2 values */

process(2);

s2_val = sigval - dks_val;

r2_val = refval - dkr_val;

printf (":led2:\%+7.5f:\%+7.5f", (float) s2_val , (float) r2_val );

sprintf( t_buf, "S2 = \%7.5f",s2_val); $/ *$ form str for lcd */

dis_lcdo;

\}

void rtemp_dis(void) $\left\{\quad f^{*}\right.$ take and display a RTD reading *f

ad_exe(Itemp);

printf (":rt:\%+7.5f", rt_val);

void es_exe (void) $\{\quad / *$ execute a sequence */

$\mathrm{RI}=0$;

while ( $R I=0$ \&\& ds $=1$ ) \{

$\mathrm{z}=1$;

while $(\mathrm{z}<=(\operatorname{len} / 3-1))$ [

if $(11==\mathrm{z})$ p1_dis();

if $(12=z)$ p2 dis 0 ;

if $(\overline{0} 1=\mathrm{z})$ otemp_dis 0 ;

if $(02=\mathrm{z})$ otemp2_dis $\mathrm{O}$;

if $(\mathrm{rt}=\mathrm{z}) \mathrm{rtemp} \operatorname{dis} \mathrm{O}$;

if ( $\mathrm{dk}==\mathrm{z}) \mathrm{dk}$ dis();

if $(\mathrm{te}==\mathrm{z}) \mathrm{T}$ _dis $\mathrm{O}$;

$\mathrm{z+t}$;

\}

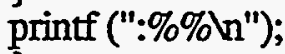

\}

void sp_exe (void) \{

printf ("pw = ");

scanf (" \%f",\&pw);

st_exe (pw);

void delay(void) \{

yy $=0$;

while $(\mathrm{yy}<10)$ yy + ;

\}

void send(void) $\{*$ routine to send 8 bits on serial bus, ack verified */

$\mathrm{i}=0$;

while $(i<8)\{$

SIBIT $=$ I2C_data;

I2C_data =_crol_(I2C_data, 1);

i+t;

\}

SDO = high; $/ *$ releases data line for memory response */

ack = S1BIT; $/ *$ sends 9 th clock pulse and looks at data line *I

delayO; 


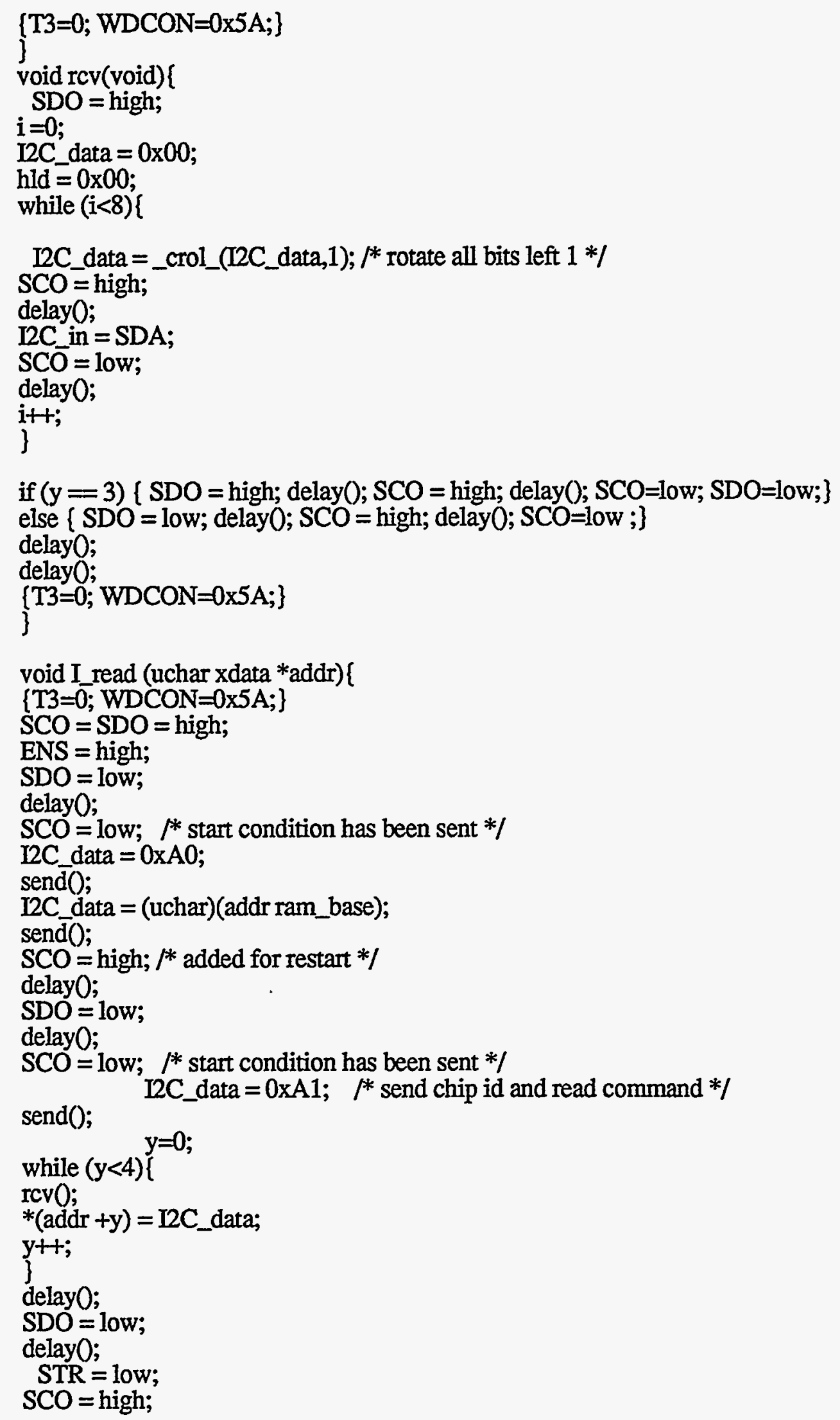




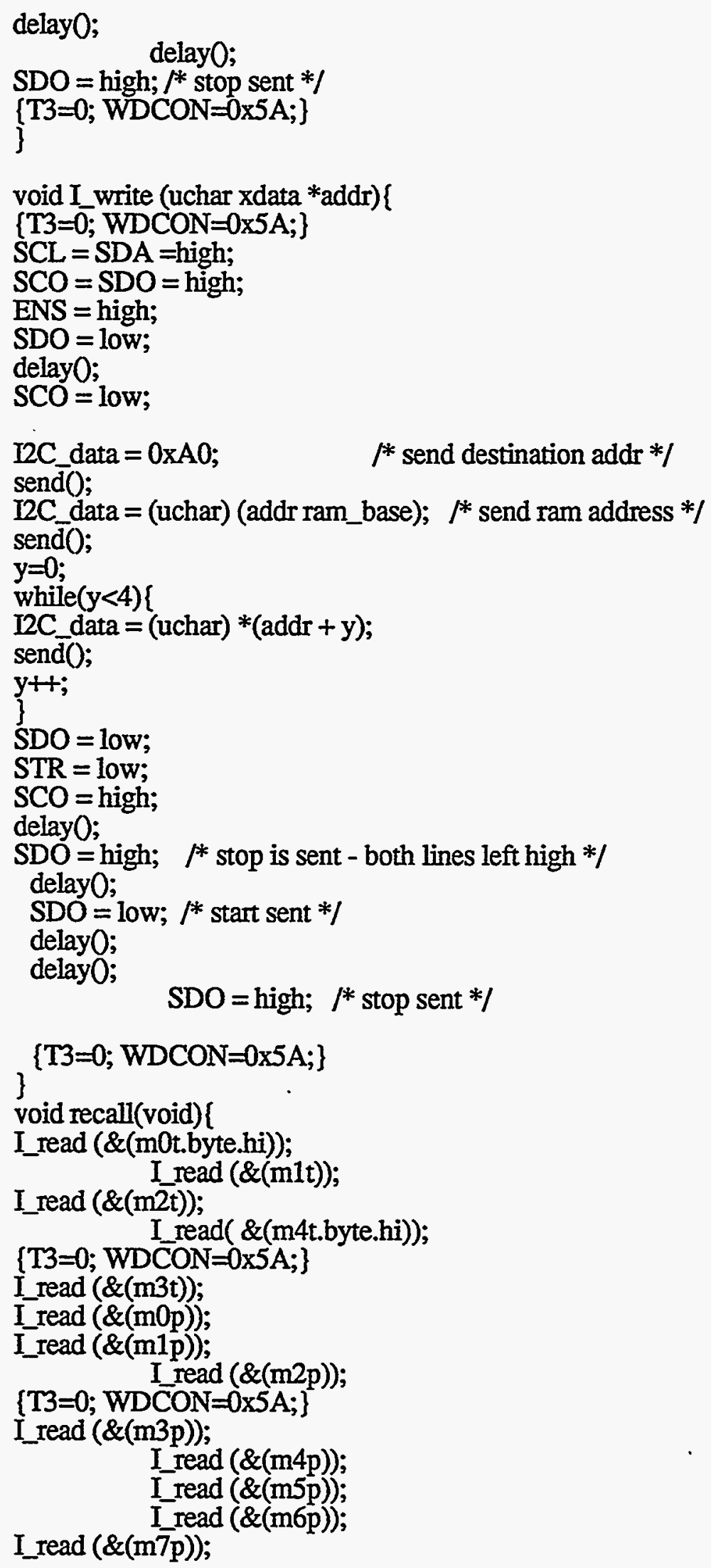


I_read (\&(m8p));

$\{\mathrm{T} 3=0 ; \mathrm{WDCON}=0 \times 5 \mathrm{~A} ;\}$

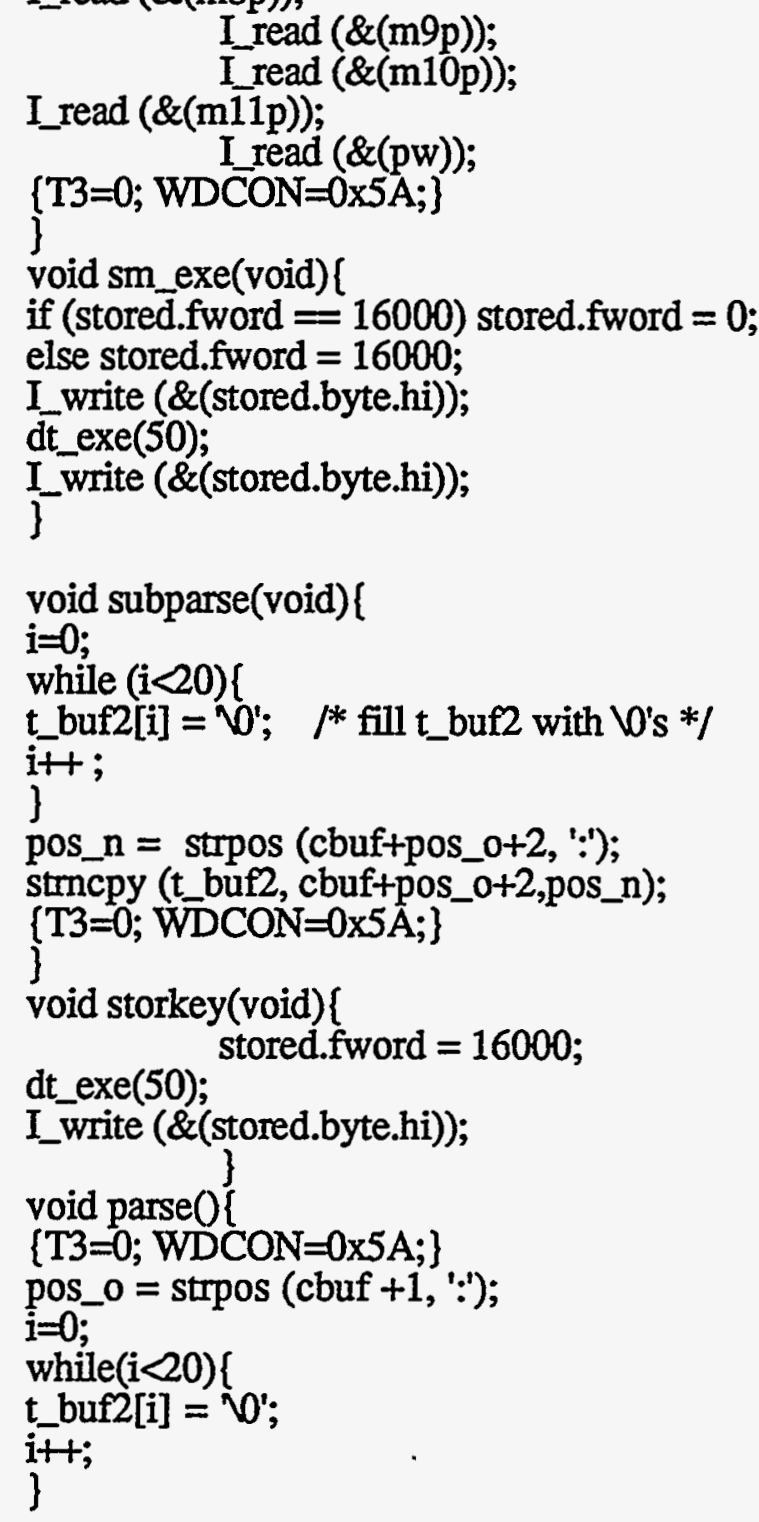

strncpy (t_buf2, cbuf+1,pos_o); $/ *$ copy first 2 digit code into cs *I

if $\left(\right.$ strcmp $\left.\left(t \_b u f 2, " d s "\right)==0\right)\left\{1 *\right.$ if $t \_b u f=$ "ds" proceed otherwise exit */

len = strlen (cbuf);

if $($ len $\% 3=1 \& \&$ len $>4)($

$\mathrm{l} 1=\mathrm{l} 2=01=02=\mathrm{rt}=\mathrm{dk}=\mathrm{te}=0$;

$\mathrm{y}=1$;

$\{\mathrm{T} 3=0 ; \mathrm{WDCON}=0 \times 5 \mathrm{~A} ;\}$

while $(\mathrm{y}<=(\operatorname{len} / 3-1))\{$

pos_n = strpos (cbuft+pos_o+2, ':');

stmcpy (t_buf2, cbuftpos_o+2,pos_n);

if (strcmp (t_buf2, "11")==0) \{ printf ("11_on:"); $11=y$; \}

if (strcmp (t_buf2, "12")=0) \{ printf ("12_on:"); $12=\mathrm{y} ;\}$

if (strcmp (t_buf2, "o1")==0) \{ printf ("ot_on:"); $01=y$; \}

if (strcmp (t_buf2, "o2") $=0$ ) $02=y$; 


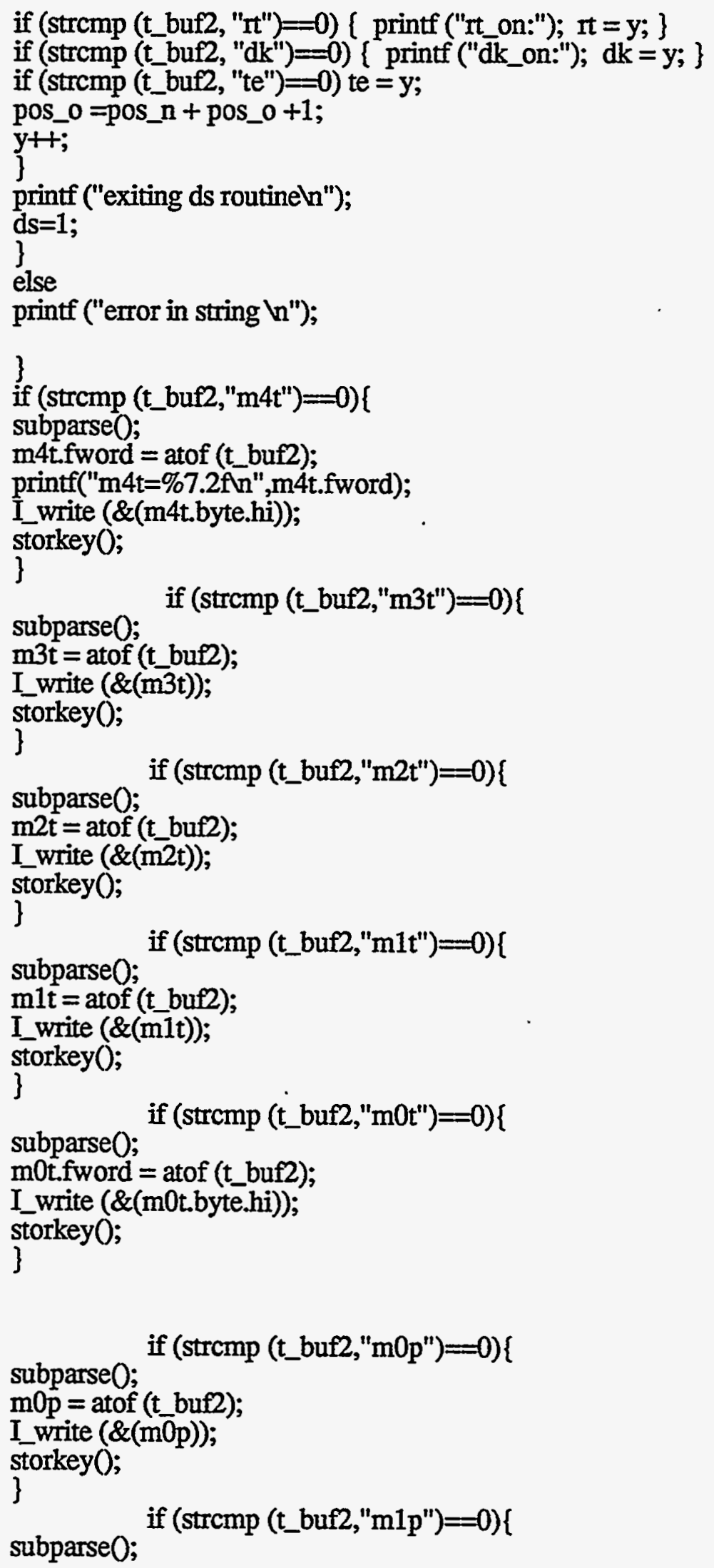


$\mathrm{m} 1 \mathrm{p}=$ atof ( $\mathrm{t}$ buf2);

I_write (\&(m1p));

storkey();

\}

subparse();

if (strcmp (t_buf2,"m2p") $=0)\{$

$\mathrm{m} 2 \mathrm{p}=$ atof ( $\mathrm{t}$ buf2);

I_write $(\&(\mathrm{~m} 2 \mathrm{p}))$;

storkey();

\}

subparse();

if (strcmp (t_buf2,"m3p")==0)\{

$\mathrm{m} 3 \mathrm{p}=$ atof ( $\mathrm{t}$ buf $)$;

I_write (\&(m3p));

storkey();

\}

subparse();

if (strcmp ( $t$ buf2,"m4p")==0)

$\mathrm{m} 4 \mathrm{p}=$ atof ( $\mathrm{t}$ buf 2$)$;

I_write (\&(m4p));

storkey();

\}

subparse);

if (strcmp (t_buf2,"m5p")=0)\{

m5p = atof (t_buf2);

I_write (\&(m5p));

storkey();

\}

subparse();

if (strcmp (t_buf2,"m6p")==0)

m6p = atof ( $\mathrm{t}$ buf2);

I_write $(\&(\mathrm{~m} 6 \mathrm{p}))$;

storkey();

]

subparse();

if (strcmp (t_buf2,"m7p")=0)

$\mathrm{m} 7 \mathrm{p}=$ atof ( $\mathrm{t}$ buf 2$)$;

I_write (\&(m7p));

storkey();

\}

subparse();

if (strcmp (t_buf2,"m8p")=0)(

$\mathrm{m} 8 \mathrm{p}=$ atof ( $\mathrm{t}$ buf 2$)$;

I_write (\&(m8p));

storkey();

\}

subparse();

if (strcmp (t_buf2,"m9p") $=0)$ (

$\mathrm{m} 9 \mathrm{p}=$ atof ( $\mathrm{t}$ buf2);

I_write (\&(m9p));

storkey 0 ;

\}

subparse();

if (strcmp (t_buf2,"m10p") $=0)\{$ 


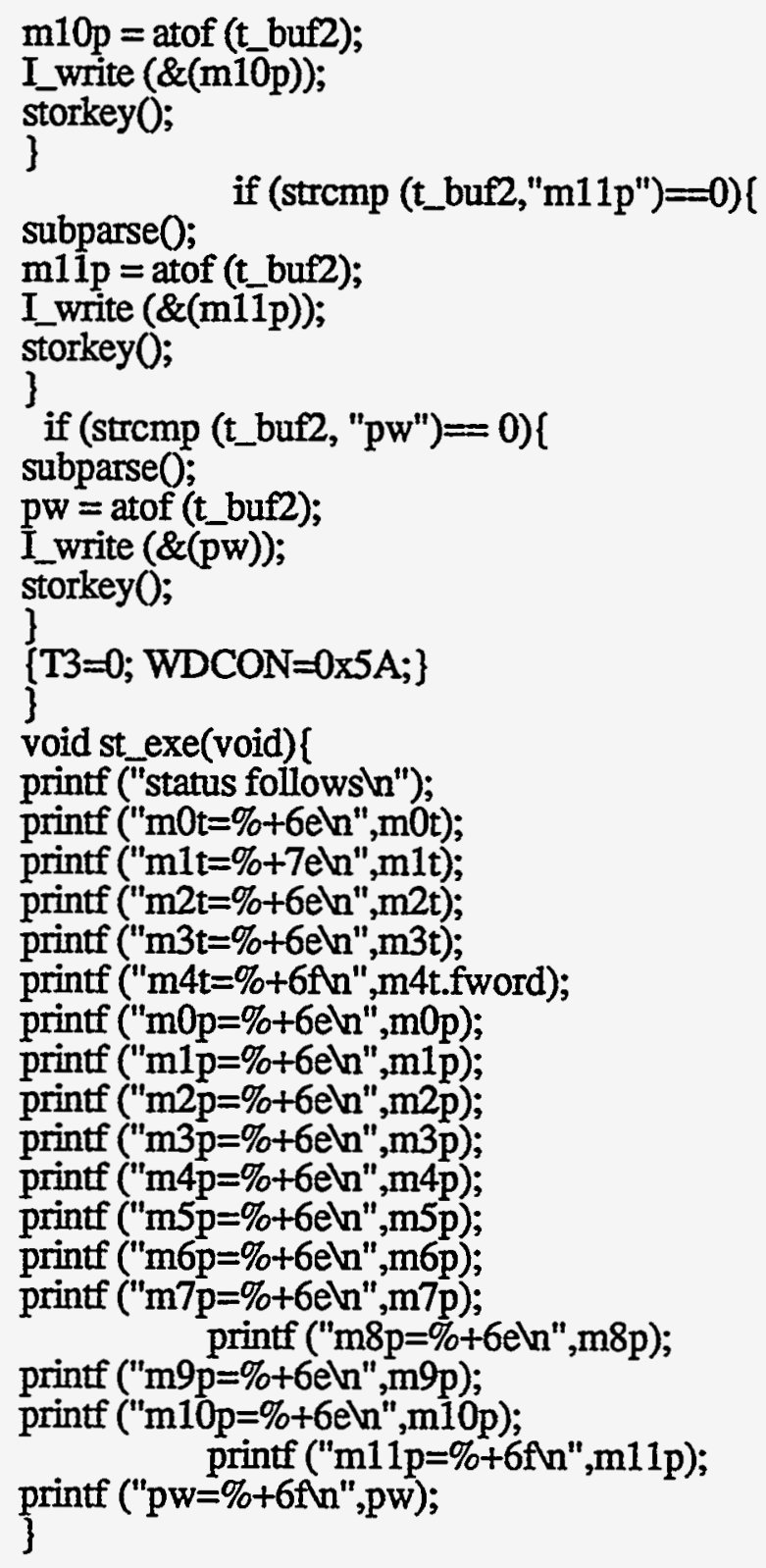


if (strcmp (cbuf , "cw") =0) printf ("\%sin",cwst);

if ( strcmp (cbuf, "11" ) ==0 ) \{p1_dis(); printf(":"); \}

if ( strcmp (cbuf, "12") $=0$ ) \{ p2_dis(); printf(":"); \}

if (strcmp (cbuf, "o1") ==0) \{ otemp_dis(); printf(":"); \}

if (strcmp (cbuf, "02" ) ==0 ) \{ otemp2_dis(); printf(":""); \}

if (strcmp (cbuf, "rt" ) $=0$ ) \{ rtemp_dis(); printf(":"); \}

if (strcmp (cbuf, "oo") ==0) o_switch(on);

if (strcmp (cbuf, "of") ==0) o_switch(off);

if (strcmp (cbuf, "da") ==0 ) DA_exe();

if (strcmp (cbuf, "te" ) =0) T_dis();

if (strcmp (cbuf, "sd") =0 ) [sw = $\sim$ sw; printf ("display switchedn"); \}

if (strcmp (cbuf, "sm" )=0) sm_exe();

if ( strcmp (cbuf, "es") $=0$ ) \{

es_exe();

$\mathrm{RI}=0$;

\}

if ( strcmp (cbuf , "st" ) =0 ) st_exe();

if ( strcmp (cbuf, "sp") ==0) sp_exe();

if (strcmp (cbuf , "wt") $=0$ ) while (1);

if ( cbuf[0] = ':' $)$ [ $/ *$ printf ("cbuf =\%s ds=\%dn",cbuf,ds); */ parse0); \}

printf (' $n$ ");

\} $/ *$ while end */

printf ("'n");

$\mathrm{RI}=0$;

\} $/ *$ command end *I

void main (void) \{

* xdata char cbuf [4]; */

$\mathrm{IE} 0=0$;

$\mathrm{SOCON}=0 \times 50$

TMOD $=0 \times 21 ; \rho^{*}$ timer 18 bit reloadable : timer 016 bit timer $* /$

$\mathrm{TCON}=0 \mathrm{xCO}$;

$\mathrm{TH} 1=0 \mathrm{xFD}$;

TR1 $=1$;

$\mathrm{b} 0=\mathrm{b} 1=0$;

led $1=$ led2 $=0$;

* $\mathrm{P} 1=0 \mathrm{x0d} ; * 1$

$P *$ IENO $=0 ; *$ I

$\mathrm{TI}=1$;

$\mathrm{RS}=\mathrm{E}=0$;

F_set0; $\quad{ }^{*}$ Icd initialization *I

D_off $0 ; *$ Icd initialization *I

mode_set $0 ; / *$ lcd initialization */

hold $=$ reset $=1$;

$\{T 3=0 ; \mathrm{WDCON}=0 \times 5 \mathrm{~A} ;\}$

$\mathrm{WDCON}=0 \times 5 \mathrm{~A}$;

$\{\mathrm{T} 3=0 ; \mathrm{WDCON}=0 \times 5 \mathrm{~A} ;\}$

S1SCS $=0 \times 02$;

$\mathrm{SCL}=\mathrm{SDA}=\mathrm{on}$;

sprintf(t_buf , "initializing");

dis_lcd0;

$\mathrm{cs}=$ sdata $=$ drdy_not $=1$;

$\mathrm{DAC}=\mathrm{DAD}=\mathrm{DA} \overline{\mathrm{L}}=\mathrm{off}$;

ds $=0$; 


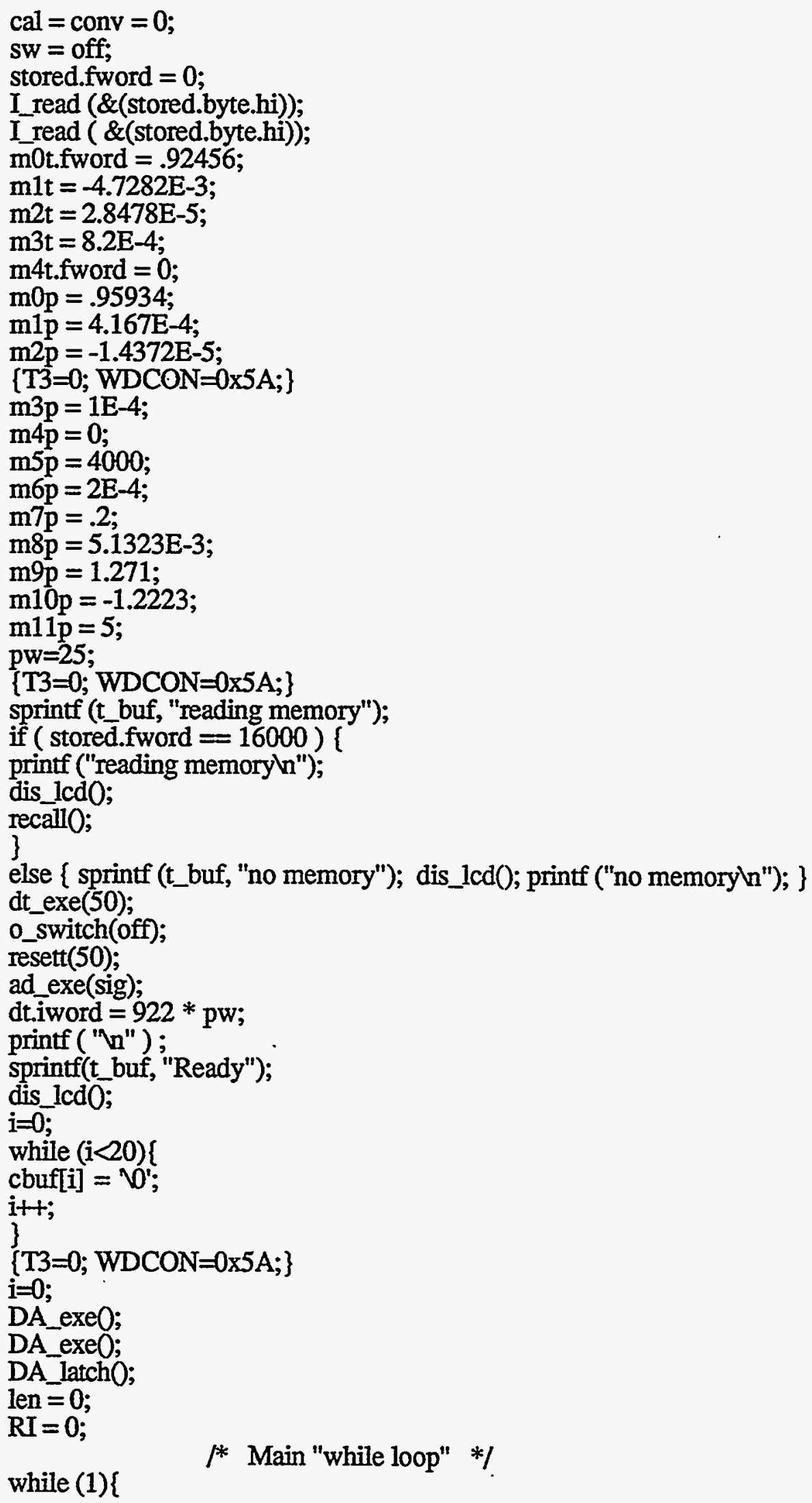




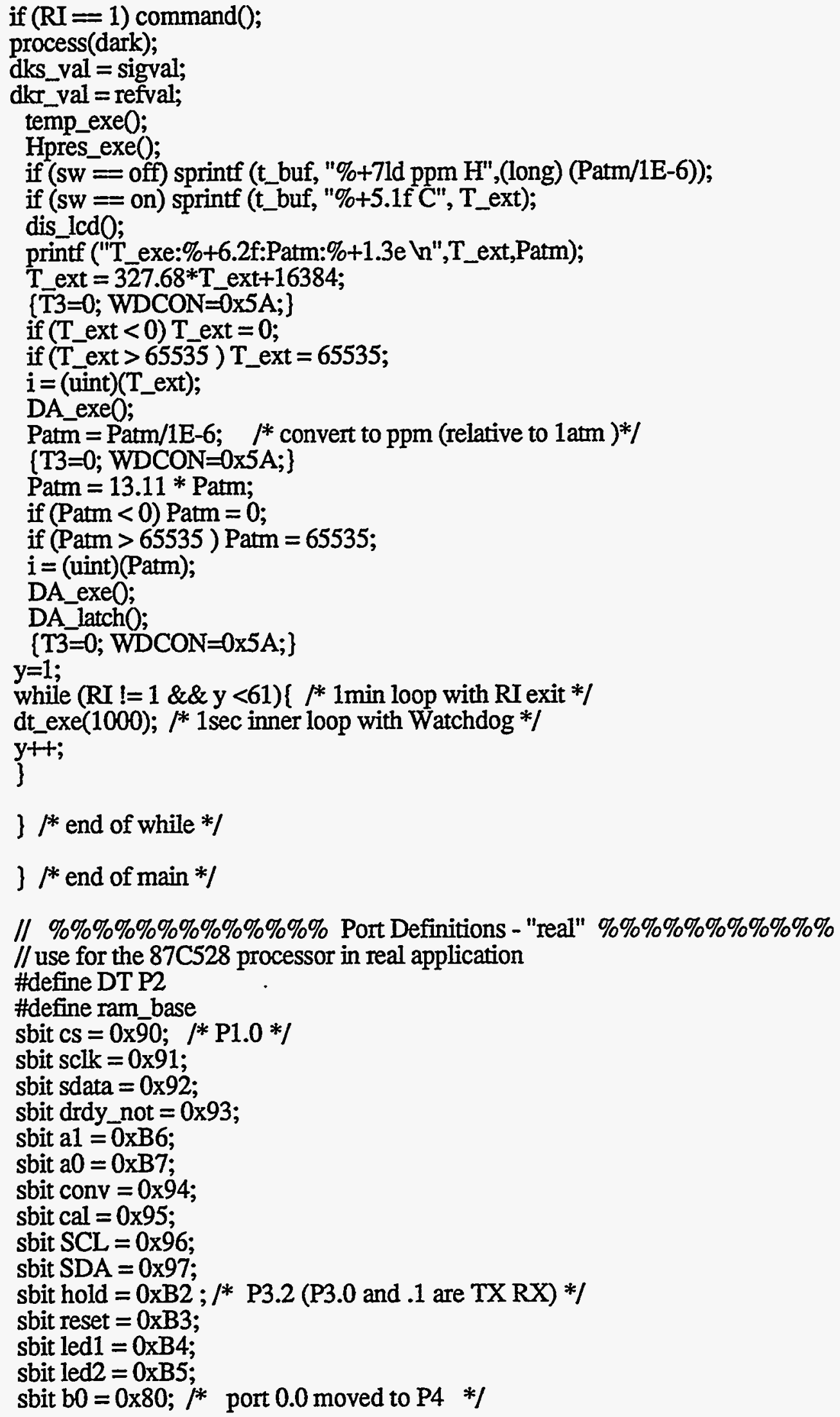


sbit $\mathrm{b} 1=0 \mathrm{x} 81 ; \rho^{*}$ port 0.1 moved to $\mathrm{P} 4$ for testing */ sbit $\mathrm{RS}=0 \times 82 ; / *$ LCD control - port $4.0 *$ / sbit $\mathrm{E}=0 \times 83 ; / *$ LCD control - port $4.2 *$ I sbit $\mathrm{DAD}=0 \times 84$

sbit $\mathrm{DAC}=0 \times \mathrm{x} 85$

sbit DAL = 0x86;

/* \%\%\%\%\%\%\%\%\% End of Port Definitions - real \%\%\%\%\%\%\%\%\%\%\% 
Distribution List

\begin{tabular}{lll} 
Copies & & MailStop \\
\hline 20 & & 1425 \\
5 & & 1425 \\
1 & 1425 \\
1 & 1425 \\
1 & 1425 \\
1 & 1425 \\
1 & 1425 \\
1 & 1074 \\
5 & 0527 \\
1 & 0527 \\
1 & 1380 \\
1 & 9018 \\
5 & 0899 \\
1 & 0619 \\
2 & 0100 \\
106 &
\end{tabular}

Addressee

M. A. Butler, 1315

R. C. Sanchez, 1315

R. C. Hughes, 1315

S. J. Martin, 1315

M. A. Mitchell, 1315

R. Thomas, 1315

K. B. Pfeifer, 1315

M. W. Scott, 1307

G. R. Dulleck, 2235

A. R. Anderson, 2235

D. Lambert, 4213

Central Technical Files, 8523-2

Technical Library, 4414

Print Media, 12615

Document Processing, 7613-2

DOE/OSTI

U.S. Department of Energy

Office of Scientific and Technical Information

Attn: Mary Beasley

Oak Ridge, TN 37830

Outside Addressees:

1
1
5

J. W. Harley

R. Berris

R. Langan

9177 Dutton Dr.

Twinsburg, OH 44087 
